\title{
MARTINGALES IN A $\sigma$-FINITE MEASURE SPACE INDEXED BY DIRECTED SETS $\left.{ }^{1}\right)$
}

\author{
BY \\ Y. S. $\left.\mathrm{CHOW}^{2}\right)$
}

Introduction. Martingale theory for a linearly ordered index set in a finite measure space has been systematically discussed by Doob [4]. As convergence theory and system theory are the two main topics in martingale theory, Helms [6] has extended Doob's results on mean convergence to martingales in a finite measure space, indexed by directed sets, and Krickeberg $[8 ; 9]$ extended the results of pointwise convergence and stochastic convergence to martingales in a $\sigma$-finite measure space, indexed by directed sets. Krickeberg uses measure algebra and lattice theoretic methods, and in [8] makes the assumption in pointwise convergence that the martingales have some covering property, which is called $V_{0}$ in his paper. Dieudonné [3] has given a martingale which is not pointwise convergent and has non-negative, bounded functions in a finite measure space, indexed by a countable directed set. Therefore, some additional conditions like Krickeberg's $V_{0}$ to the usual ones seem to be necessary for pointwise convergence. However, the condition $V_{0}$ is too weak to be the only additional condition needed for pointwise convergence of martingales indexed by noncountable directed sets. Some conditions such as terminal separability are needed.

For the system theory, Bochner [2] has given some extensions to martingales indexed by directed sets, but without proofs. Some of his results are, unfortunately, not true. Snell [14] has defined regularity (which is closely related to system theorems) of martingales in a finite measure space, indexed by positive integers, and has given some sufficient conditions. He has not discussed the necessary conditions, nor the relation between regularity and the system theorems.

In this paper, $\$ 1$ gives preliminary definitions and notation. $\$ 2$ is devoted to the definition of conditional expectation in a $\sigma$-finite measure space $W$, and $\S 3$ to that of martingales indexed by directed sets in $W$ as well as to individual system theorems and inequalities. In $\$ 4$, a general pointwise convergence theorem is proved, and a new kind of convergence theorem, based on a differentiation theorem of interval functions [13, p. 192] is given. We

Presented to the Society, January 22, 1959 under the title Pointwise convergence of martingales with a directed index set; received by the editors March 24, 1959.

(1) This work was supported in part by the National Science Foundation under Grant G-2794. The results were submitted to the University of Illinois in partial fulfillment of the requirements for the Ph.D. degree. The author wishes to express his appreciation to Professor J. L. Doob who supervised the preparation of the thesis.

(2) Now with IBM Research Center, Yorktown Heights, New York. 
give three Vitali conditions; one of them is the same as Krickeberg's and another one is strong enough to guarantee the pointwise convergence of martingales under the usual conditions. $\$ 5$ is devoted to the differentiation theory of an interval function in a Euclidean space. In that section the usual Lebesgue theorem is proved as an application of previous convergence theorems. An extension of Andersen and Jessen's result [1] on countably additive set functions to finitely additive set functions is given in $\$ 7$.

1. Definitions and notation. In the following $(W, \mathfrak{F}, \mu)$ will be a fixed $\sigma$-finite measure space, where $W$ is an abstract set with points $w, \mathfrak{F}$ is a $\sigma$-algebra of subsets of $W, \mu$ is a $\sigma$-finite complete measure on $W$. A function will be extended real valued, if we do not mention the range explicitly. If $x$ is a function defined on a subset $W^{\prime}$ of $W, \max (x, 0)$ and $\max (-x, 0)$ will be respectively denoted by $x^{+}$and $x^{-}$. The integral $\int_{V} x d \mu$ will be shortened to $\int_{V} x$, if no confusion arises.

If $B$ is a family of sets, we will use the terms (S)-sets, (B)-measurable, and (B)-functions respectively for sets belonging to $\mathbb{G}$, measurable with respect to (\$), and functions being (\$-measurable. An $\mathfrak{F}$-set is called a null set if it is of measure 0 . The empty set is denoted by $\varnothing . B(B)$ will be the $\sigma$-algebra generated by $(\$)$, i.e., the smallest $\sigma$-algebra containing (\$).

Let $x$ be an $\mathfrak{F}$-function defined on a subset $W^{\prime}$ of $W$, and $\mathbb{H}$ be a family of $\mathfrak{F}$-sets containing $W^{\prime}$. If either $\int_{V} x^{+}$or $\int_{V} x^{-}$defines a $\sigma$-finite measure on (B) in $W^{\prime}$, then $x$ is said to be $\sigma$-integrable on $\mathcal{S}$ in $W^{\prime}$. If $x$ is $\sigma$-integrable on $\mathfrak{F}$ in $W$, we will just say that $x$ is $\sigma$-integrable. If $\int_{W^{\prime}} x^{+}$or $\int_{W^{\prime}} x^{-}$is finite, $x$ is said to be integrable in $W^{\prime}$. If $x$ is integrable in $W$, we will just say $x$ is integrable. Replacing "or" by "and" in the above two definitions, we will have the definitions for $x$ to be $\sigma$-summable and summable respectively. If $x$ is integrable, we will write $E(x)=\int_{W} x$.

If $f$ is a function defined in $W^{\prime} \subset W$ with the range $R^{\prime}$, the set " $\left\{w: x(w) \in R R^{\prime}\right\}$ " will be denoted by $\{x \in R\}$. The set of all positive integers is denoted by $N$, and $0 \cdot \infty$ will be defined as 0 . "Countable" will mean denumerable or finite. Let $A$ and $B$ be two sets. " $A-B$ " and " $\chi_{A}$ " will mean respectively $A-A B$ and the characteristic function of $A, \bar{\mu}$ will be the outer measure induced by $\mu$. It is easy to prove that:

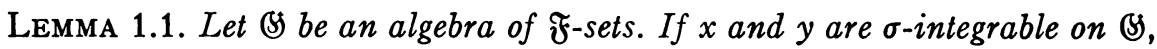
then there exists a sequence $U_{1} \subset U_{2} \subset U_{3} \subset \ldots$ of $\$$-sets such that $\lim U_{n}=W$ and both $x$ and $y$ are integrable in each $U_{n}$. If $x$ and $y$ are $\sigma$-integrable, $\sigma$-summable, or summable on $\$$, so is $\max (x, y)$.

Let $\&$ be an algebra of $\mathfrak{F}$-sets. A $B$-set of positive measure is said to be a (S)-atom if $\mu(A-U)=0$ for every (S)-set $U \subset A$ of positive measure. Two atoms $A$ and $A^{\prime}$ are considered to be the same if $\mu\left(A-A^{\prime}\right)+\mu\left(A^{\prime}-A\right)=0$.

2. Conditional expectations.

Definition 2.1. Let $\mathbb{B}$ be a $\sigma$-algebra of $\mathfrak{F}$-sets. If the restriction of $\mu$ to 
$\mathfrak{W}$ is $\sigma$-finite, then $\mathbb{B}$ is said to be $\sigma$-finite.

Let $x$ be $\sigma$-integrable on a $\sigma$-finite $\sigma$-algebra \& of $\mathfrak{F}$-sets, and let $U_{1} \subset U_{2} \subset U_{3} \subset \cdots$ be a sequence of (S)-sets such that $\lim U_{n}=W$ and $x$ is integrable in each $U_{n}$. Let $\mathfrak{S}_{n}=\left(V: V \in \mathcal{G}, V \subset U_{n}\right)$. Then $\mathscr{S}_{n}$ is a $\sigma$-algebra. By the Radon-Nikodym theorem $\left[5 ;\right.$ p. 131], there exists a $\mathscr{S}_{n}$-measurable function $x_{n}$ (which may have value $\pm \infty$ ) in $U_{n}$ such that, for every $\mathbb{S}_{n}$-set $V$,

$$
\int_{V} x=\int_{V} x_{n} .
$$

For $w \in U_{k}-U_{k-1}$, where $U_{0}=\varnothing$, define

$$
E\left(x \mid(\xi)(w)=x_{k}(w)\right. \text {. }
$$

Evidently, $E(x \mid$ ()) is $\&$-measurable and for every $(S-$-set $V$ in which $x$ is integrable,

$$
\int_{V} E\left(x \mid \text { (j) }=\int_{V} x .\right.
$$

Let $y$ be any (\$)-function satisfying (2.3), by replacing $E(x \mid$ (s) by $y$. Then $y=E(x \mid$ (S) a.e. by the uniqueness of the Radon-Nikodym derivatives on each $U_{n}$. Thus, a $(S$-function $E(x \mid$ (S) is uniquely (up to an equivalence) defined by (2.3). $E(x \mid(S)$ is said to be the conditional expectation of $x$ given $\$$. An equivalent definition of $E(x \mid \mathbb{S})$ has been given in [7]. From the definition, we have immediately:

Corollary 2.1. Let \&S be a $\sigma$-finite $\sigma$-algebra of $\mathfrak{F}$-sets and let $x$ and $y$ be $\sigma$-integrable on $\$$. If there is a sequence $U_{1} \subset U_{2} \subset U_{3} \subset \cdots$ of $(\$-$ sets such that $\lim U_{n}=W$ and both $x$ and $y$ are integrable in $U_{n}$, and if for each $U_{n}$ and each (\$)-set $V \subset U_{n} \int_{V} x \leqq \int_{V} y$, then $E(x \mid$ (\$) $\leqq E(y \mid$ (\$) a.e.

Let $x$ and $y$ be $\sigma$-integrable on a $\sigma$-algebra of $\mathfrak{F}$-sets. Then by following the corresponding results of [4], we have:

(2.4) $E(x \mid$ () $\geqq 0$ a.e. if $x \geqq 0$ a.e.; $E(1 \mid$ () $)=1$ a.e.;

(2.5) $E(c x \mid$ (S) $=c E(x \mid$ (S) a.e. if $c$ is a real number;

(2.6) $E^{+}\left(x \mid\right.$ (S) $\leqq E\left(x^{+} \mid\right.$(S) and $E^{-}\left(x \mid\right.$ (S) $\leqq E\left(x^{-} \mid\right.$(\$) a.e.;

(2.7) $E\left(x+y \mid\right.$ (S) $=E\left(x \mid\right.$ (S) $+E\left(y \mid\right.$ (S) a.e. if $x^{+}$and $y^{+}$are $\sigma$-summable, or if $x^{-}$and $y^{-}$are $\sigma$-summable.

(2.8) If $y$ is a real-valued $\$$-function, then

$$
E(x y \mid \text { (S) }=y E(x \mid \text { (\$) a.e. }
$$

(2.9) If $\left(y_{n}, N\right)$ is a sequence of $\mathfrak{F}$-functions such that $\lim y_{n}=y$ a.e., and if $x$ is $\sigma$-summable on $\&$ such that $\left|y_{n}\right| \leqq x$ a.e. for every $n$, then,

$$
\lim E\left(y_{n} \mid \text { (s) }=E(y \mid \text { (s) a.e. }\right.
$$


(2.10) Let $\mathscr{S S} \subset \mathbb{B}^{\prime}$ be $\sigma$-finite, $\sigma$-algebras of $\mathfrak{F}$-sets and $x \sigma$-integrable on (3. Then $E\left(x \mid \mathcal{S}^{\prime}\right)$ is $\sigma$-integrable on (B). If, moreover, $x$ is integrable, so is $E(x \mid$ (S).

(2.11) Let $\$ S \subset\left(S^{\prime}\right.$ be $\sigma$-finite, $\sigma$-algebras of $\mathfrak{F}$-sets and $x \sigma$-integrable on (5. Then,

$$
E\left(E\left(x \mid \text { (S) } \mid \mathfrak{B}^{\prime}\right)=E\left(x \mid \text { (S) }=E\left(E\left(x \mid \mathbb{S}^{\prime}\right) \mid\right. \text { (S). a.e. }\right.\right.
$$

THEOREM 2.1. Let $\$$ be a $\sigma$-finite $\sigma$-algebra of $\mathfrak{F}$-sets, $x$ and $y$ be $\sigma$-summable on (S), $x$ be (S-measurable, and $g$ be a real-valued, continuous, convex function on the real line such that $\mathrm{g}^{+}(y)$ is $\sigma$-summable on (S). If (i) $x=E(y \mid$ (S) a.e., or (ii) $g$ is nondecreasing and $x \leqq E(y \mid$ (j) a.e., then $g(x) \leqq E(g(y) \mid$ (j) a.e.

Proof. Since $g$ is continuous and convex, the right-hand side derivative $g_{+}^{\prime}(t)$ of $g$ at $t$ exists and is finite for every real $t$, and $g_{+}^{\prime}$ is nondecreasing and

$$
g\left(t^{\prime}\right)-g(t) \geqq g_{+}^{\prime}(t)\left(t^{\prime}-t\right)
$$

for every real $t^{\prime}$. Therefore, $g_{+}^{\prime}(x)$ is $(5$-measurable. By Lemma 1.1, there exist (\$-sets $U_{1} \subset U_{2} \subset U_{3} \subset \ldots$ such that $x, y$ and $g^{\prime}(y)$ are summable in each $U_{n}$ and $\lim U_{n}=W$. Let $V$ be a $\left(\xi\right.$-set and $V \subset U_{n}$. Let $x=E(y \mid$ (S) a.e. For $k \in N$, put $V_{k}=V\left\{\left|g_{+}^{\prime}(x)\right| \leqq k\right\}$. Then $g_{+}^{\prime}(x)(y-x)$ is summable in $V_{k}$ and

$$
\begin{aligned}
\int_{V_{k}} g_{+}^{\prime}(x)(y-x) & =\int_{V_{k}} E\left(g_{+}^{\prime}(x)(y-x) \mid \text { (S) }\right) \\
& =\int_{V_{k}} g_{+}{ }^{\prime}(x)(E(y \mid \text { (S) }-x)=0 .
\end{aligned}
$$

Since $g_{+}(x)(y-x)$ is summable in $V_{k}, g(y)-g(x)$ is integrable in $V_{k}$ by (2.12), and by (2.12) and (2.13)

$$
\int_{V_{2}}[g(y)-g(x)] \geqq 0 .
$$

If $V=\{g(x)>0\} U_{n}$, then (2.14) implies that

$$
\int_{U_{n}\left\{0(x)>0,\left|\sigma^{\prime}+(x)\right| \leqslant k\right\}}\left[g^{+}(y)-g^{+}(x)\right] \geqq \int_{U_{n}\left\{\theta(x)>0,\left|\sigma^{\prime}+(x)\right| \leq k\right\}}[g(y)-g(x)] \geqq 0 .
$$

Therefore,

$$
\infty>\int_{U_{n}} g^{+}(y) \geqq \int_{\left.U_{n}|| \theta^{\prime}+(x) \mid \leq k\right\}} g^{+}(x)
$$

Letting $k \rightarrow \infty$ we have $\int_{U_{n}} g^{+}(x)<\infty$. By (2.14),

$$
\int_{V_{k}} g(y) \geqq \int_{V_{k}} g(x)
$$


for every (S)-set $V \subset U_{n}$, where $V_{k}=V\left\{\left|g_{+}^{\prime}(x)\right| \leqq k\right\}$. Letting $k \rightarrow \infty$, we have $\int_{V} g(y) \geqq \int_{V} g(x)$. Therefore, $E(g(y) \mid$ (3) $\geqq E(g(x) \mid$ (j) $=g(x)$ a.e., since $g(x)$ is (5)-measurable.

For (ii), $g(x) \leqq g(E(y \mid())) \leqq E(g(y) \mid$ (S) a.e. by (i). The proof is complete.

When $g(y)$ is $\sigma$-summable on $(\mathcal{B}$, Theorem 2.1 has been proved by Doob [ 4 ; p. 295]. (2.9) can not be improved in the following sense. For the Lebesgue measure on the interval $[0,1)$, and for any extended real number $a \geqq 0$, there always exists a sequence of Lebesgue measurable functions $\left(x_{n}, N\right)$ such that $\lim x_{n}=0, x_{n} \geqq 0, \lim E\left(x_{n}\right)=0$, and

$\lim \sup E\left(x_{n} \mid\right.$ (S) $=a, \quad \lim \inf E\left(x_{n} \mid\right.$ (S) $=0$, a.e. for some $\$$.

To see this, for any $k \in N$ and $k \leqq 4^{n}$, we write $k$ as

$$
k=\left(i_{1}-1\right) 4^{n-1}+\left(i_{2}-1\right) 4^{n-2}+\cdots+\left(i_{n-1}-1\right) 4+i_{n},
$$

where $i_{1}, i_{2}, \cdots, i_{n}=1,2,3,4$. Put $\left.I_{i_{1}, i_{2}}, \cdots i_{n}=\left[(k-1) / 4^{n}, k / 4^{n}\right)\right]$, where $k=1,2, \cdots, 4^{n}, n=1,2, \cdots$. Let

$$
\begin{aligned}
& U_{1}^{1}=I_{1} \cup I_{3}, U_{2}^{1}=I_{2} \cup I_{4}, \\
& U_{1}^{2}=I_{1,1} \cup I_{1,3} \cup I_{3,1} \cup I_{3,3}, \quad U_{2}^{2}=I_{1,2} \cup I_{1,4} \cup I_{3,2} \cup I_{3,4}, \\
& U_{3}^{2}=I_{2,1} \cup I_{2,3} \cup I_{4,1} \cup I_{4,3}, \quad U_{4}^{2}=I_{2,2} \cup I_{2,4} \cup I_{4,2} \cup I_{4,4} \\
& U_{1}^{3}=I_{1,1,1} \cup I_{1,1,3} \cup I_{1,3,1} \cup I_{1,3,3} \cup I_{3,1,1} \cup I_{3,1,3} \cup I_{3,3,1} \cup I_{3,3,3}, \\
& U_{2}^{3}=I_{1,1,2} \cup I_{1,1,4} \cup I_{1,3,2} \cup I_{1,3,4} \cup I_{3,1,2} \cup I_{3,1,4} \cup I_{3,3,2} \cup I_{3,3,4}, \cdots .
\end{aligned}
$$

Suppose that $(5)$ is the algebra of sets generated by the $U$ 's. For any given extended real number $a \geqq 0$, take a sequence of real numbers $\left(a_{n}, N\right)$ such that $\lim a_{n}=a$. Define $\left(x_{n}, N\right)$ as follows:

$$
\begin{aligned}
& x_{1}=2 a_{1} \chi_{I_{1}}, x_{2}=2 a_{2} \chi_{I_{2}}, x_{3}=4 a_{3} \chi_{I_{1,1},}, x_{4}=4 a_{4} \chi_{I_{1,2},}, x_{5}=4 a_{5} \chi_{I_{2,1},}, \\
& x_{6}=4 a_{6} \chi_{I_{2,2}}, x_{7}=8 a_{7} \chi_{I_{1,1,1}}, x_{8}=8 a_{8} \chi_{I_{1,1,2}}, \cdots,
\end{aligned}
$$

then $\lim x_{n}=0$ and $\lim E\left(x_{n}\right)=0$. But lim $\sup E\left(x_{n} \mid\right.$ (S) $=a$ and $\lim \inf E\left(x_{n} \mid\right.$ (S) $=0$ a.e., since $E\left(x_{1} \mid\right.$ (S) $=a_{1} \chi_{U_{1}^{1}}^{1}, E\left(x_{2} \mid\right.$ (S) $)=a_{2} \chi_{U_{2}^{1}}, E\left(x_{3} \mid\right.$ (S) $=a_{3} \chi_{U_{1}^{2}}, E\left(x_{4} \mid\right.$ (S) $=a_{4} \chi_{U_{2}^{2}}^{2}, E\left(x_{5} \mid\right.$ (S) $=a_{5} \chi_{U_{3}^{2}}^{2}, E\left(x_{6} \mid\right.$ (S) $=a_{6} \chi_{U_{4}^{2}}^{2}, E\left(x_{7} \mid\right.$ S) $=a_{7} \chi_{U_{1}^{3}}^{3}, \quad E\left(x_{8} \mid\right.$ (S) $=a_{8} \chi_{U_{2}}{ }^{3}, \cdots$.

\section{Martingales.}

Definition 3.1. A stochastic basis is a net $\left(\mathfrak{F}_{\delta}, \Delta\right)$, where $\Delta$ is a directed

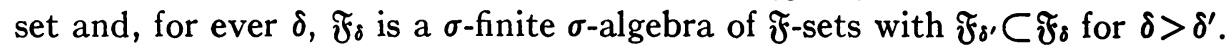
A stochastic process is a triple $\left(x_{\delta}, \mathfrak{F}_{\delta}, \Delta\right)$, where $\left(\mathfrak{F}_{\delta}, \Delta\right)$ is a stochastic basis and $x_{\delta}$ is $\mathfrak{F}_{\delta}$-measurable for each $\delta$.

For a stochastic process $\left(x_{\delta}, \Delta\right)$ we define

$$
\lim \sup x_{\delta}=\inf _{\delta} \sup _{\delta^{\prime} \geqq \delta} x_{\delta^{\prime}}, \quad \liminf x_{\delta}=\sup _{\delta} \inf _{\delta^{\prime} \geqq \delta} x_{\delta^{\prime}},
$$

where $\delta$ and $\delta^{\prime} \in \Delta$. If no confusion arises, we will write 


$$
\bar{x}=\limsup _{\delta} x_{\delta} \quad \text { and } \quad \bar{x}=\lim _{\delta} \inf x_{\delta}
$$

When $\bar{x}=\tilde{x}$, we write $\lim x_{\delta}=\bar{x}$.

Definition 3.2. A stochastic process $\left(x_{\delta}, \Delta\right)$ is said to be terminally separable, if there exists a countable cofinal subset $\Delta^{\prime}$ of $\Delta$ such that $\bar{x}=\lim \sup _{\delta \in \Delta^{\prime}} x_{\delta}$ and $\tilde{x}=\lim \inf _{\delta \in \Delta^{\prime}} x_{\delta}$ a.e.

Definition 3.3. A function $s$ defined on $W$ with countable range $\Delta^{\prime} \subset \Delta^{\prime \prime}$, where $\Delta^{\prime \prime}$ is a directed set including $\Delta$ as a sub-directed set, is called a stopping variable of a stochastic process $\left(x_{\delta}, \mathfrak{F}_{\delta}, \Delta\right)$ or a stochastic basis $\left(\mathfrak{F}_{\delta}, \Delta\right)$, if $\{s \leqq \delta\} \in F_{\delta}$ and $\{s \geqq \delta\} \in \mathfrak{F}_{\delta}$ for each $\delta$. A stopping variable is called a sampling variable if $\Delta^{\prime} \subset \Delta$. A net $\left(s_{\lambda}, \Lambda\right)$ of sampling variables is called a sampling process if $s_{\lambda} \leqq s_{\lambda}$ a.e. for $\lambda \leqq \lambda^{\prime}$.

It is easy to see that, if $s$ is a stopping variable, all the sets $\{s=\delta\}$, $\{s>\delta\},\{s<\delta\},\{s \leq \delta\},\{s \geq \delta\} ;$ and $\{s \pm$ or $\$ \delta\} \in \mathfrak{F}_{\delta}$ for each $\delta \in \Delta$.

Let $s$ be a sampling variable of a stochastic process $\left(x_{\delta}, \mathfrak{F}_{\delta}, \Delta\right)$. We define a new $\mathfrak{F}$-function $x^{*}$ on the set $\{s \in \Delta\}$ by

$$
x^{*}(w)=x_{s(w)}(w) .
$$

$x^{*}$ is sometimes denoted by $x_{*}$ also. If $\left(s_{\lambda}, \Lambda\right)$ is a sampling process, and $\left(\mathfrak{F}_{\delta}, \Delta\right)$, we define

$$
x_{\lambda}^{*}=x_{\lambda_{\lambda}} \text {. }
$$

THEOREM 3.1. Let $\left(x_{\delta}, \mathfrak{F}_{\delta}, \Delta\right)$ be a stochastic process, and $\left(s_{\lambda}, \Lambda\right)$ be a sampling process of $\left(\mathfrak{F}_{\delta}, \Delta\right)$. Let

$$
\mathfrak{F}_{\lambda}^{*}=\mathbb{B}\left\{V\left\{s_{\lambda} \geqq \delta\right\} ; \delta \in \Delta, V \in \mathfrak{F}_{\delta}\right\} .
$$

Then $\left(x^{*}, \mathfrak{F}_{\lambda}^{*}, \Lambda\right)$ is a stochastic process and

$$
\mathfrak{F}_{\lambda}^{*}=\mathbb{B}\left\{V\left\{s_{\lambda}=\delta\right\} ; \delta \in \Delta, V \in \mathfrak{F}_{\delta}\right\} \text {. }
$$

Proof. Evidently we may assume that $\Delta$ is countable. For any real $a$ and any $\delta \in \Delta$,

$$
\left\{x_{\lambda}^{*} \geqq a\right\}\left\{s_{\lambda}=\delta\right\}=\left\{x_{\delta} \geqq a, s_{\delta}=\delta\right\} \in F_{\delta} .
$$

Hence, $\left\{x_{\lambda}^{*} \geqq a, s_{\lambda}=\delta\right\}=\left\{x_{\delta}^{*} \geqq a, s_{\lambda}=\delta\right\}\left\{s_{\lambda} \geqq \delta\right\} \in F_{\lambda}^{*}$. Therefore, $\left\{x_{\lambda}^{*} \geqq a\right\}$ $\in \mathfrak{F}_{\lambda}^{*}$ and $x_{\lambda}^{*}$ is $\mathfrak{F}_{\lambda}^{*}$-measurable.

To prove the $\mathfrak{F}_{\lambda}^{*} \subset F_{\lambda^{\prime}}^{*}$ for $\lambda<\lambda^{\prime}$, let $V \in F_{\delta}$. Then $V\left\{s_{\lambda} \geqq \delta\right\} \in F_{\delta}, V\left\{s_{\lambda} \geqq \delta\right\}$ $=V\left\{s_{\lambda} \geqq \delta, s_{\lambda^{\prime}} \geqq \delta\right\} \in F_{\lambda}^{*}$, and therefore $F_{\lambda}^{*} \subset F_{\lambda^{\prime}}^{*}$.

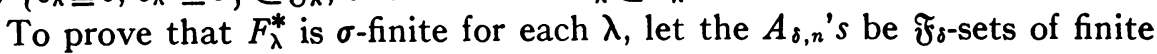
measures and $\bigcup_{n=1}^{\infty} A_{\delta, n}=W$ for each $\delta$. Then

$$
W=\bigcup_{\delta \in \Delta}\left\{s_{\lambda} \geqq \delta\right\}=\bigcup_{\delta \in \Delta} \bigcup_{n=1}^{\infty}\left\{s_{\lambda} \geqq \delta\right\} A_{\delta, n} .
$$


Since $\mu\left\{s_{\lambda} \geqq \delta\right\} A_{\delta, n}<\infty, \mathfrak{F}_{\lambda}^{*}$ is $\sigma$-finite. Thus $\left(x_{\lambda}^{*}, \mathfrak{F}_{\lambda}^{*}, \Lambda\right)$ is a stochastic process.

To prove (3.4), let $\mathfrak{F}_{\lambda}^{\prime}=\circledast\left[V\left\{s_{\lambda}=\delta\right\}: \delta \in \Delta, V \in \mathfrak{F}_{\delta}\right]$. Then $V\left\{s_{\lambda} \geqq \delta\right\}$ $=U_{\delta^{\prime} \geq \delta} V\left\{s_{\lambda}=\delta^{\prime}\right\} \in F_{\lambda^{\prime}}$, if $V \in \mathfrak{F}_{\delta}$. Therefore, $\mathfrak{F}_{\lambda}^{*} \subset F_{\lambda}^{\prime}$. Now $V\left\{s_{\lambda}=\delta\right\}$ $=V\left\{s_{\lambda}=\delta, s_{\lambda} \geqq \delta\right\} \in \mathfrak{F}_{\lambda}^{*}$, if $V \in \mathfrak{F}_{\delta}$. Thus $\mathfrak{F}_{\lambda}^{\prime} \subset \mathfrak{F}_{\lambda}^{*}$. Hence $\mathfrak{F}_{\lambda}^{*}=\mathfrak{F}_{\lambda}^{\prime}$.

CoROllaRy 3.1. Under the conditions of Theorem 3.1, if $V^{*} \in F_{\lambda}^{*}$ then $V^{*}\left\{s_{\lambda}=\delta\right\} \in F_{\delta}$ for each $\delta$.

Proof. Let $\mathfrak{A}=\left\{A: A \in \mathfrak{F}_{\lambda}^{*}, A\left\{s_{\lambda}=\delta\right\} \in \mathfrak{F}_{\delta}\right.$ for some $\left.\delta\right\}$. Then $V\left\{s_{\lambda}=\delta\right\} \in \mathfrak{A}$ for $V \in \mathfrak{F}_{8}$, and if $A \in \mathfrak{A}$, then $W-A \in \mathfrak{A}$. Let $A_{1} \subset A_{2} \subset A_{3} \subset \cdots$ and $A_{n} \in \mathfrak{A}$. Then $\lim A_{n} \in \mathfrak{A}$. Therefore, $\mathfrak{A}=\mathfrak{F}_{\lambda}^{*}$.

Definition 3.4. A stochastic process $\left(x_{\delta}, \mathfrak{F}_{\delta}, \Delta\right)$ is a semi-martingale if $x_{\delta}$ is $\sigma$-integrable on every $\mathfrak{F}_{\delta^{\prime}}$ for each $\delta$ and $E\left(x_{\delta} \mid \mathfrak{F}_{\delta^{\prime}}\right) \geqq x_{\delta^{\prime}}$ a.e. for $\delta^{\prime}<\delta$. If, moreover, for each $\delta, x_{\delta}$ is $\sigma$-summable on every $\mathfrak{F}_{\delta^{\prime}}, x_{\delta}$ is integrable, or $x_{\delta}$ is summable, then $\left(x_{\delta}, \mathfrak{F}_{\delta}, \Delta\right)$ is said to be a $\sigma$-summable, an integrable, or a summable semi-martingale, respectively. If sup $_{\delta \in \Delta} \delta \in \Delta$, the semi-martingale $\left(x_{\delta}, \mathfrak{F}_{\delta}, \Delta\right)$ is said to be closed. If the inequality is replaced by an equality, we have the corresponding definitions for martingales.

TheOREM 3.2. Let $\left(x_{\delta}, \mathfrak{F}_{\delta}, \Delta\right)$ and $\left(y_{\delta}, \mathfrak{F}_{\delta}, \Delta\right)$ be two semi-martingales, and let $z_{\delta}=\max \left(x_{\delta}, y_{\delta}\right)$. Then $\left(z_{\delta}, \mathfrak{F}_{\delta}, \Delta\right)$ is a semi-martingale.

Proof. By Lemma 1.1, for each $\delta^{\prime} \in \Delta, z_{\delta}$ is $\sigma$-integrable on $\mathfrak{F}_{\delta^{\prime}}$, since $x_{\delta}$ and $y_{\delta}$ are, and for each. $\delta^{\prime}<\delta$ there exists a sequence $U_{1} \subset U_{2} \subset U_{3} \subset \ldots$ of $\mathfrak{F}_{\delta^{\prime}}$-sets such that $x_{\delta}, y_{\delta}$ and $z_{\delta}$ are integrable in each $U_{n}$ and $\lim U_{n}=W$. Let $V$ be an $\mathfrak{F}_{\delta^{\prime}}$-set and $V \subset U_{n}$, then

$$
\begin{aligned}
\int_{V} z_{\delta^{\prime}} & =\int_{V\left\{x_{\left.\delta^{\prime} \leq y_{\delta^{\prime}}\right\}}\right.} y_{\delta^{\prime}}+\int_{V\left\{x_{\delta^{\prime}}>y_{\delta^{\prime}}\right\}} x_{\delta^{\prime}} y_{\delta}+\int_{V\left\{x_{\delta^{\prime}}>y_{\delta^{\prime}}\right\}} x_{\delta} \\
& \leqq \int_{V\left\{x_{\left.\delta^{\prime} \leq y_{\delta^{\prime}}\right\}}\right.} z_{\delta}+\int_{V\left\{x_{\delta^{\prime}}>y_{\delta^{\prime}}\right\}} z_{\delta}=\int_{V} z_{\delta}=\int_{V} E\left(z_{\delta} \mid F_{\delta^{\prime}}\right) .
\end{aligned}
$$

By Corollary $2.1, z_{\delta^{\prime}}=E\left(x_{\delta} \mid \mathfrak{F}_{\delta^{\prime}}\right)$ a.e. if $\delta^{\prime}<\delta$, and $\left(z_{\delta}, \mathfrak{F}_{\delta}, \Delta\right)$ is a semimartingale.

We have the following corollary immediately:

CoRollary 3.2. If $\left(x_{\delta}, \mathfrak{F}_{\delta}, \Delta\right)$ is a semi-martingale, so is $\left(x_{\delta}^{+}, \mathfrak{F}_{\delta}, \Delta\right)$. If $\left(x_{\delta}, \mathfrak{F}_{\delta}, \Delta\right)$ is a martingale, then $\left(\left|x_{\delta}\right|, \mathfrak{f}_{\delta}, \Delta\right)$ is a semi-martingale.

When $\left(x_{\delta}, \mathfrak{F}_{\delta}, \Delta\right)$ is $\sigma$-summable, this corollary can also be obtained from Theorem 2.1, since $x^{+}$and $|x|$ are continuous convex functions of $x$ and $x^{+}$ is nondecreasing. In the following, $N^{*}$ and $R$ will be the set of all integers and rational numbers respectively. 
TheOREM 3.3. Let $\left(x_{n}, \mathfrak{F}_{n}, N^{*}\right)$ be a semi-martingale and $\left(s_{n}, N^{*}\right)$ a sampling process of $\left(\mathfrak{F}_{n}, N^{*}\right)$. If for each $n$,

$x_{n}^{*}$ is $\sigma$-integrable on every $\mathfrak{F}_{k}^{*}$, and

$$
\liminf _{k \rightarrow \infty} \int_{\left\{o_{n}>k\right\}} x_{k}^{+}=0
$$

for each $n$, then $\left(x_{n}^{*}, \mathfrak{F}_{n}^{*}, N^{*}\right)$ is a semi-martingale.

When $\mu(W)<\infty$ and $\left(x_{n}, \mathfrak{F}_{n}, N\right)$ is a summable semi-martingale, Theorem 3.3 has been proved by Doob [4, p. 302]. By a slight modification, his proof can be used to prove this theorem.

CoROllaRy 3.3. Let $\left(x_{n}, \mathfrak{F}_{n}, N^{*}\right)$ be a semi-martingale and $\left(s_{n}, N^{*}\right)$ a sampling process. If $s_{n}$ has only finitely many values for each $n$, and $x_{n}^{+}$is $\sigma$-summable for every $n$ on every $\mathfrak{F}_{k}$ or $x^{-}$is $\sigma$-summable for every $n$ on every $\mathfrak{F}_{k}$, then $\left(x_{n}^{*}, \mathfrak{F}_{n}^{*}, N^{*}\right)$ is a semi-martingale.

Lemma 3.1. Let $\left(x_{r}, \mathfrak{F}_{r}, R\right)$ be a semi-martingale such that $x_{r} \geqq 0$, and for every sampling variable $s$

$$
\underset{r}{\lim \sup } \int_{\{0>r\}} x_{r}<\infty
$$

Then,

$$
\lim _{r} \int_{[0>r]} x_{s}=0
$$

If, moreover, $E\left(x_{r}\right)<\infty$ for each $r$, then $E\left(x_{s}\right)<\infty$ for every sampling variable $s$.

Proof. If (3.8) is false, there exist a positive $d$, a sampling variable $s$, and a sequence $r_{1}<r_{2}<r_{3}<\cdots$ of $R$ such that $\int\left(r_{i+1} z_{8}>r_{i}\right) x_{8}>i d$. Let $r^{\prime \prime}>r^{\prime}$; then

$$
\int_{\left\{r^{\prime \prime} \geq 8>r^{\prime}\right\}} x_{8}=\sum_{r^{\prime}<r \leq r^{\prime \prime}} \int_{\{8=r\}} x_{r} \leqq \sum_{r^{\prime}<r \leq r^{\prime \prime}} \int_{\{8=r\}} x_{r^{\prime \prime}}=\int_{\left\{r^{\prime \prime} \geq 8>r^{\prime}\right\}} x_{r^{\prime \prime}}
$$

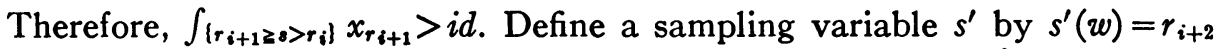

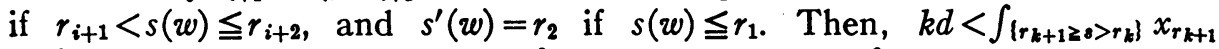
$=\int_{\left\{z^{\prime}-r_{k+2}\right]} x_{r_{k+1}}$. Thus, $\lim \sup _{r} \int_{\left\{\varepsilon^{\prime}>r\right\}} x_{r} \geqq \lim \sup _{k \rightarrow \infty} \int_{\left\{\varepsilon^{\prime}>r_{k+1}\right]} x_{r_{k+1}}=\infty$, and this contradicts (3.7). Hence, (3.8) holds. To prove the second part, we have $\int_{\{s \leq r\}} x_{s} \leqq \int_{(s \leq r\}} x_{r}$ from (3.9), and then

$$
E\left(x_{8}\right)=\int_{\{8 \leqq r\}} x_{r}+\int_{\{8>r\}} x_{8}<\infty,
$$

if $r$ is large enough. The proof is complete. 
LEMMA 3.2. Let $\left(x_{n}, \mathfrak{F}_{n}, N\right)$ be a semi-martingale and $E\left(x_{n}^{-}\right)<\infty$ for each $n$. Then $E\left(x_{s}^{-}\right)<\infty$ for every sampling variable $s$, if for every sampling variable $s$

$$
\lim \sup \int_{(s>n)} x_{n}^{+}<\infty .
$$

Proof. For a given sampling variable $s$, put $A_{n}=\{s=n\}$ and $A_{n}^{\prime}=\{s>\grave{n}\}$. By Lemma 3.1 and (3.10), we can take $n \in N$ and $k \in N$ such that $\int_{A_{n}^{\prime}} x_{z}^{+}<k$ and $\int_{\Lambda_{i^{\prime}}} x_{i}^{+}<k$ whenever $i \geqq n$. Then, for $j>n$,

$$
\begin{aligned}
\int_{A^{\prime} n} x_{n} & =\int_{A_{n+1}} x_{n}+\int_{A_{n+1}^{\prime}} x_{n} \leqq \int_{A_{n+1}} x_{n+1}+\int_{A_{n+1}^{\prime}} x_{n+1} \leqq \cdots \\
& \leqq \sum_{i=n+1}^{j} \int_{A_{i}} x_{i}+\int_{A^{\prime} j} x_{j} \leqq \sum_{i=n+1}^{j}\left(\int_{A_{i}} x_{i}^{+}-\int_{A_{i}} x_{i}^{-}\right)+\int_{A^{\prime} j} x_{j}^{+} .
\end{aligned}
$$

Therefore,

$$
\begin{aligned}
\sum_{i=n+1}^{j} \int_{A_{i}} x_{i}^{-} & \leqq-\int_{A^{\prime}{ }_{n}} x_{n}+\sum_{i=n+1}^{j} \int_{A_{i}} x_{i}^{+}+\int_{A^{\prime} j} x_{j}^{+} \\
& \leqq \int_{A^{\prime}{ }_{n}} x_{n}^{-}+\int_{A_{n}^{\prime}{ }_{n}} x_{i}^{+}+\int_{A^{\prime} j} x_{j}^{+}
\end{aligned}
$$

Hence, $\int\left\{j z_{8}>n\right\} x_{s}^{-} \leqq \int_{A_{n}^{\prime}} x_{n}^{-}+2 k$ for every $j>n$. Thus, $\int_{A_{n}^{\prime}} x_{s}^{-}<\infty$, and then $E\left(x_{z}^{-}\right)<\infty$, since $\int_{(8 \leq n)} x_{z}^{-} \leqq \sum_{i=1}^{n} \int_{W} x_{n}^{-}<\infty$.

LeMma 3.3. Let $\left(x_{n}, \mathfrak{F}_{n}, N\right)$ be a martingale and $x_{1}^{+}$be $\sigma$-summable on $\mathfrak{F}_{1}$. Suppose that $s$ is a sampling variable of $\left(\mathfrak{F}_{n}, N\right)$ and

$$
\lim _{n} \int_{\{0>n\}} x_{i}^{+}=0
$$

Then $x_{s}^{+}$is $\sigma$-summable on $\mathfrak{F}_{1}$.

Proof. Take $k \in N$ so large such that $\int_{(8>k)} x_{s}^{+}<1$. By Lemma 1.1, there are $\mathfrak{F}_{1 \text {-sets }} U_{1} \subset U_{2} \subset U_{3} \cdots$ such that $\lim U_{n}=W$ and all the $x_{i}$ 's, $i \leqq k$, are integrable and $x_{1}^{+}$is summable in each $U_{n}$. If $i \leqq k$, then $\int_{U_{n}} x_{i}=\int_{U_{n}} x_{1} \leqq \int_{U_{n}} x_{1}^{+}$ $<\infty$. Therefore, $x_{i}^{+}$is also summable in $U_{n}$ for $i \leqq k$. Hence,

$$
\int_{U_{n}\{e \leq k\}} x_{i}^{+} \leqq \sum_{i=1}^{k} \int_{U_{n}} x_{i}^{+}<\infty .
$$

Hence, $x_{s}^{+}$is summable in $U_{n}$ and then $\sigma$-summable on $\mathfrak{F}_{1}$.

TheOREM 3.4. Let $\left(s_{\lambda}, \Lambda\right)$ be a sampling process of a martingale $\left(x_{\delta}, \mathfrak{F}_{\delta}, \Delta\right)$ and $\Delta^{\prime \prime}$ be a cofinal subset of $\Delta$. Suppose that

(3.13) for each $\lambda, x_{\lambda}^{*}$ is $\sigma$-integrable on every $\mathfrak{F}_{\lambda^{\prime}}^{*}, \lambda^{\prime} \in \Lambda$, and 
(3.14) for each countable subset $\Delta^{\prime}=\left(\delta_{n}, n=1,2,3 \cdots\right)$ of $\Delta^{\prime \prime}$ with $\delta_{1}<\delta_{2}<\delta_{3}<\cdots$, and every sampling variable $s^{\prime}$ of $\left(\mathfrak{F}_{\delta}, \Delta^{\prime}\right)$,

$$
\limsup _{n \rightarrow \infty} \int_{\left\{\varepsilon^{\prime}>\delta_{n}\right\}}\left|x_{\delta_{n}}\right|<\infty
$$

and

$$
\underset{n \rightarrow \infty}{\liminf } \int_{\left\{\varepsilon^{\prime}>\delta_{n}\right\}}\left|x_{\delta_{n}}\right|=0
$$

or

$(3.14)^{\prime}$ each $s_{\lambda}$ has only finitely many values. Then $\left(x_{\lambda}^{*}, \mathfrak{F}_{\lambda}^{*}, \Lambda\right)$ is a martingale.

Proof. Let $\lambda, \lambda^{\prime} \in \Lambda$ and $\lambda<\lambda^{\prime}$. By (3.13), $x_{\lambda}^{*}$ and $x_{\lambda^{\prime}}^{*}$ are $\sigma$-integrable on $\mathfrak{F}_{\lambda}^{*}$, there are $\mathfrak{F}_{\lambda}^{*}$-sets $U_{1} \subset U_{2} \subset U_{3} \subset \ldots$ such that $\lim U_{n}=W$ and both $x_{\lambda}^{*}$ and $x_{\lambda^{\prime}}^{*}$ are integrable in every $U_{n}$, by Lemma 1.1. To prove $E\left(x_{\lambda^{\prime}}^{*} \mid \mathfrak{F}_{\lambda}^{*}\right)=x_{\lambda}^{*}$ a.e., we need only to prove that, by Corollary 2.1 , for each $U_{n}$ and each $\mathfrak{F}_{\lambda}^{*}$-set $U \subset U_{n}$,

$$
\int_{U} x_{\lambda}^{*}=\int_{U}^{x_{\lambda^{\prime}}^{*}}
$$

Since $s_{\lambda}$ has only countably many values and both $x_{\lambda}^{*}$ and $x_{\lambda}^{*}$, are integrable in $U$, it suffices to prove that, for $V=U\left\{s_{\lambda}=\delta\right\}$,

$$
\int_{V} x_{\lambda}^{*}=\int_{V} x_{\lambda^{\prime}}^{*}
$$

Let all the values $\delta^{\prime}$ of $s_{\lambda^{\prime}}$ satisfying $\delta^{\prime}>\delta$ be the set $\left(\delta_{n}, N^{\prime}\right)$, where $N^{\prime}=(1,2,3, \cdots)$ is finite or infinite. Then $(3.16)$ is equivalent to

$$
\int_{V} x_{\delta}=\sum_{n \in N^{\prime}} \int_{V^{\prime}\left\{\delta \lambda^{\prime}=\delta_{n}\right\}} x_{\delta_{n}}
$$

Let $\delta_{1}^{\prime} \geqq \delta_{1}$ and $\delta_{1}^{\prime} \in \Delta^{\prime \prime}$, and when the first $j$ indices $\delta_{1}^{\prime} \leqq \delta_{2}^{\prime} \leqq \cdots \leqq \delta_{j}^{\prime}$, $j \in N^{\prime}$, have been defined, we denote by $\delta_{j+1}^{\prime}$ any index of $\delta$ of $\Delta^{\prime \prime}$ such that $\delta>\delta_{j}^{\prime}$ and $\delta>\delta_{j}$ if $j+1 \in N^{\prime}$, and stop the induction if $j+1 \notin N^{\prime}$. Then we have a subset $\left(\delta_{n}^{\prime}, N^{\prime}\right)$ of $\Delta^{\prime \prime}$ such that $\delta<\delta_{1}^{\prime} \leqq \delta_{2}^{\prime} \leqq \cdots$ and $\delta_{n}<\delta_{n}^{\prime}$ for each $n \in N^{\prime}$.

Let $n_{1}=1$ and $n_{k+1}$ be the smallest $j \in N^{\prime}$ such that $\delta_{j}^{\prime}>\delta_{n_{k}}^{\prime}$ if there is one, for $k=1,2,3, \cdots$ (finite or infinite), and stop the induction if else. For each $k$ let $\delta_{n k}^{\prime}=\delta_{k}^{\prime \prime}$ and let the set of $\delta^{\prime \prime}$ 's be $\left(\delta_{k}^{\prime \prime}, N^{\prime \prime}\right)$, where $N^{\prime \prime}=(1,2,3, \cdots)$ (finite or infinite). Then $N^{\prime \prime} \subset N^{\prime}, \delta<\delta_{1}^{\prime \prime}<\delta_{2}^{\prime \prime}<\cdots$, and $\delta_{k}^{\prime \prime} \geqq \delta_{j}^{\prime} \geqq \delta_{j}$ for $j<n_{k+1}$.

Define $s^{\prime}(w)=\delta$. If $s_{\lambda^{\prime}}(w) \geq \delta$, and $s^{\prime}(w)=\delta_{k}^{\prime \prime}$ if $s_{\lambda^{\prime}}(w)=\delta_{j}$, for $j \in N^{\prime}$, 
$n_{k} \leqq j<n_{k+1}$. (If $n_{k+1}$ is not defined, $n_{k} \leqq j<n_{k+1}$ is interpreted as $n_{k} \leqq j$.) Then

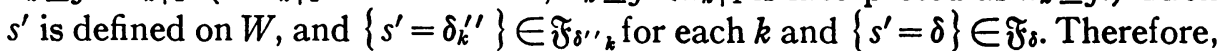
$s^{\prime}$ is a sampling variable of $\left(\delta, \delta_{1}^{\prime \prime}, \delta_{2}^{\prime \prime}, \delta_{3}^{\prime \prime}, \cdots\right)$ and $s^{\prime}>\delta$. Each of (3.14) and (3.14)' implies that $x_{8}$ is $\sigma$-integrable on $\mathfrak{F}_{\delta}$ by Lemmas 3.1 and 3.2. From Theorem 3.3, $\int_{V} x_{\delta}=\int_{V} x_{\delta^{\prime}}$. Hence,

$$
\begin{aligned}
\sum_{j \in N^{\prime}} \int_{V\left\{\delta \lambda^{\prime}=\delta_{j}\right\}} x_{\delta_{j}} & =\sum_{k} \sum_{j \in N^{\prime}, n_{k} \leq j<n_{k+1}} \int_{V\left\{\delta \lambda^{\prime}=\delta_{j}\right\}} x_{\delta_{j}} \\
& =\sum_{k} \sum_{j \in N^{\prime}, n_{k} \leq j<n_{k+1}} \int_{V\left\{\delta \lambda^{\prime}=\delta_{j}\right\}} x_{\delta^{\prime \prime}{ }_{k}} \\
& =\sum_{k} \int_{V\left\{\delta^{\prime}=\delta^{\prime \prime}{ }_{k}\right\}} x_{\delta^{\prime \prime}{ }_{k}}=\sum_{k} \int_{V\left\{\delta^{\prime}=\delta^{\prime \prime}{ }_{k}\right\}} x_{\boldsymbol{g}^{\prime}}=\int_{V} x_{\boldsymbol{g}^{\prime}}=\int_{V} x_{\delta} .
\end{aligned}
$$

Thus (3.17) is true and $\left(x_{\lambda}^{*}, \mathfrak{F}_{\lambda}^{*}, \Lambda\right)$ is a martingale.

THEOREM 3.5. Let $\left(x_{\delta}, \mathfrak{F}_{\delta}, \Delta\right)$ be a martingale, and $\left(s_{\lambda}, \Lambda\right)$ be a sampling process. If each $s_{\lambda}$ has only finitely many values and, for each $\delta, x_{\delta}^{+}$is $\sigma$-summable on every $\mathfrak{F}_{\delta^{\prime}}, \delta^{\prime} \in \Delta$, then each $x_{\lambda}^{*+}$ is $\sigma$-summable on every $\mathfrak{F}_{\lambda^{\prime}}^{*}, \lambda^{\prime} \in \Lambda$, and therefore $\left(x_{\lambda}^{*}, \mathfrak{F}_{\lambda}^{*}, \Lambda\right)$ is a martingale.

Proof. By Theorem 3.4 we need only to prove that $x_{\lambda}^{*+}$ is $\sigma$-summable on every $\mathfrak{F}_{\lambda}^{*}$, for $\lambda^{\prime} \in \Lambda$. Let $\delta_{1}, \cdots, \delta_{k}$ be the values of $s_{\lambda}$ and $\delta_{1}^{\prime}, \cdots, \delta_{k^{\prime}}$ be those of $s_{\lambda^{\prime}}$. Since $x_{\delta_{1}}^{+}, \cdots, x_{\delta_{k}}^{+}$are $\sigma$-summable on $\mathfrak{F}_{\delta_{i^{\prime}}}$ for $i=1, \cdots, k^{\prime}$. By Lemma 1.1 there exist $\mathfrak{F}_{\delta_{i}^{\prime}}$-sets $U_{i, 1} \subset U_{i, 2} \subset U_{i, 3} \subset \cdots$ such that $\lim _{n} U_{i, n}$ $=W$, for $i=1, \cdots, k^{\prime}$, and all the $x_{\delta_{j}}^{+}, j=1, \cdots, k$, are summable in every $U_{i, n}$. Hence, $x_{\Omega_{\lambda}}^{+}$is summable in every $U_{i, n}$. Now

$$
W=\bigcup_{i=1}^{k^{\prime}}\left\{s_{\lambda^{\prime}}=\delta_{i}^{\prime}\right\}=\bigcup_{i=1}^{k^{\prime}} \bigcup_{n=1}^{\infty}\left\{s_{\lambda^{\prime}}=\delta_{i}^{\prime}\right\} U_{i, n} .
$$

Let $V_{n}=U_{s-1}^{x^{\prime}}\left\{s_{\lambda^{\prime}}=\delta_{i}^{\prime}\right\} U_{i, n}$, then $V_{n} \in F_{\lambda^{\prime}}^{*} ; \lim V_{n}=W$; and $x_{s \lambda}^{+}$is summable in every $V_{n}$, since it is summable in every $U_{i, n}$. Therefore, $x_{\lambda}^{*+}$ is $\sigma$-summable on each $\mathfrak{F}_{\lambda}^{*}$.

Similar results of Theorems 3.4 and 3.5 for semi-martingales are stated in a recent paper of Bochner [2], but, unfortunately, they are not true. For example, let $W=[0,1), \mathfrak{F}_{1}=(W, \phi), \mathfrak{F}_{2}=\mathbb{B}[[0,1 / 2),[1 / 2,1)], \mathfrak{F}_{2^{\prime}}=\mathfrak{F}_{2}=\mathfrak{F}_{3}$, and let $\mu$ be the Lebesgue measure on $W$. Define $x_{1}=0, x_{2}(w)=1$, if $w \in[0,1 / 2)$ and $x_{2}(w)=-1$ if $w \in[1 / 2,1) ; x_{2^{\prime}}=-x_{2}$ : and $x_{3}=1$. Then $\left(x_{\delta}, \mathfrak{F}_{\delta}, \Delta\right)$ is a summable semi-martingale where $\Delta=\left(1,2,2^{\prime}, 3\right)$ and the order " $<$ " of $\Delta$ is defined by $1<2<3$ and $1<2^{\prime}<3$. Define $s_{1}=1 ; s_{2}(w)=2$ for $w \in[1 / 2,1)$, and $s_{2}(w)=2^{\prime}$ for $w \in[0,1 / 2)$. Then $s_{1}$ and $s_{2}$ are sampling variables and $s_{1}<s_{2}$. But $x_{1}^{*}$ and $x_{2}^{*}$ do not form a semi-martingale since

$$
E\left(x_{1}^{*}\right)=0>-1=E\left(x_{2}^{*}\right) .
$$


THEOREM 3.6. Let $\left(x_{n}, \mathfrak{F}_{n}, k \geqq n \geqq 1\right)$ be an integrable semi-martingale and let $A=\left\{\max x_{n}>\alpha, k \geqq n \geqq l\right\}$ and $B=\left\{\min x_{n} \leqq \alpha, k \geqq n \geqq l\right\}$ where $\alpha$ is real and $l \geqq 1$. Then

$$
\begin{aligned}
& \int_{A} x_{k} \geqq \alpha \mu(A), \\
& \int_{A} x_{k} \geqq \int_{A} x_{1} .
\end{aligned}
$$

If, moreover, $E\left(x_{1}\right)$ is finite, then

$$
\alpha \mu(B) \geqq E\left(x_{1}\right)-\int_{W-B} x_{k} .
$$

Proof. Since

$$
\begin{aligned}
& \int_{\left\{x_{k}>0\right\}} x_{n} \leqq \int_{\left\{x_{k}>0\right\}} x_{n}^{+} \leqq E\left(x_{k}^{+}\right) \int_{\left\{x_{k}>0\right\}} x_{k}, \\
& \int_{\left\{x_{k}>\alpha\right\}} x_{n} \leqq \int_{\left\{x_{k}>\alpha\right\}} x_{k} .
\end{aligned}
$$

Let $\beta$ be a real number and define $y_{n}=\max \left(x_{n}, \beta\right)$. Then $\left(y_{n}, \mathfrak{F}_{n}, k \geqq n \geqq 1\right)$ is an integrable semi-martingale. Let $A_{\beta}=\left\{\max y_{n}>\alpha, k \geqq n \geqq l\right\}$. Define $s(w)=n$ to be the smallest $n$ such that $y_{n}(w)>\alpha, l \leqq n \leqq k$, and $s(w)=k$ if there is no such $n$. Then $\left(y_{1}, y_{8}\right)$ is an integrable semi-martingale. By (3.21),

$$
\int_{A_{\beta}} y_{1}=\int_{\left\{y_{8}>\alpha\right\}} y_{1} \leqq \int_{\left\{y_{8}>\alpha\right\}} y_{s}=\int_{A_{\beta}} y_{s} \leqq \int_{\{y>\alpha\}} y_{k}=\int_{A_{\beta}} y_{k},
$$

therefore

$$
\int_{A_{\beta}} y_{k} \geqq \alpha \mu\left(A_{\beta}\right), \quad \int_{A_{\beta}} y_{k} \geqq \int_{A_{\beta}} y_{1} .
$$

Let $\beta \rightarrow-\infty$, we have (3.18) and (3.19). To prove (3.20), let $s(w)=n$ be the first $n$ such that $x_{n}(w) \leqq \alpha, k \geqq n \geqq l$, if ever; $s(w)=k$, if there is no such $n$. Then $s$ is a sampling variable and $s \geqq 1$. Since $E\left(x_{1}\right)>-\infty, E\left(x_{n}\right)>-\infty$ for each $n$, and then $E\left(x_{s}^{-}\right)<\infty$. By Corollary 3.3, $E\left(x_{s}\right) \geqq E\left(x_{1}\right)$. Hence

$$
\alpha \mu(B)+\int_{W-B} x_{k} \geqq \sum_{n=z l}^{k-1} \int_{\{s=n\}} x_{n}+\int_{\{s=k\}} x_{k}=E\left(x_{s}\right) \geqq E\left(x_{1}\right) .
$$

Thus the proof is complete.

Theorem 3.6 has been proved by Doob $[4$, p. 314] for the case $\mu(W)<\infty$, $l=1$ and $\left(x_{n}, \mathfrak{F}_{n}, k \geqq n \geqq 1\right)$ is summable. It cannot be extended to martingales 
indexed by a directed set without more conditions. In fact, let $W=[0,1)$, $\mathfrak{F}=$ the class of Lebesgue measurable sets, and $\mu=$ the Lebesgue measure in $W$. Let $\Delta=\left(1,2,2^{\prime}, 3\right)$ and the order " $<$ " of $\Delta$ be defined by $1<2<3$ and

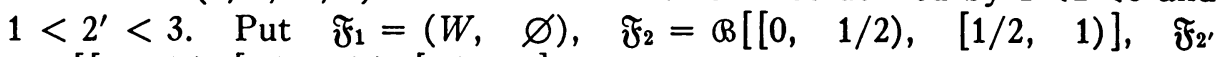
$=\bigotimes[[0,1 / 3),[1 / 3,1 / 6),[5 / 6,1)]$, and $\mathfrak{F}_{3}=\mathscr{B}\left(\mathfrak{F}_{2^{\prime}} \cup \mathfrak{F}_{2}\right)$. Define $x_{1}=1 / 2$; and $x_{2}(w)=1$ if $w \in[0,1 / 2),=0$ otherwise; $x_{2^{\prime}}(w)=1$ if $w \in[1 / 3,5 / 6),=0$ otherwise; $x_{3}(w)=3$ if $w \in[1 / 3,1 / 2)$, $=0$ otherwise. Then $\left(x_{\delta}, \mathfrak{F}_{\delta}, \Delta\right)$ is a martingale, but

$$
\mu\left\{\max x_{\delta} \geqq 1, \delta \in \Delta\right\}=5 / 6>\int_{\left\{\max x_{\delta} \geq 1, \delta \in \Delta\right\}} x_{3} .
$$

TheOREM 3.7. Let $\left(x_{n}, \mathfrak{F}_{n}, N^{*}\right)$ be a semi-martingale and $s$ be a stopping variable of $\left(\mathfrak{F}_{n}, N^{*}\right)$. If $\sup _{n \in N^{*}} E\left(\left|x_{n}\right|\right) \leqq K$, then

$$
\int_{\left(s \in N^{*}\right)}\left|x_{s}\right| \leqq 3 K \text {. }
$$

Proof. Let $x^{\prime}(w)=x_{s(w)}(w)$ if $s(w) \in N^{*}$, and $x^{\prime}(w)=0$ if $s(w) \notin N^{*}$. Put $s_{n}=\min (s, n)$. Then $\lim x_{s_{n}} \chi_{\left\{s \in N^{*}\right\}}=x^{\prime}=x_{s} \chi_{\left\{s \in N^{*}\right\}}$. In view of Fatou's theorem, it now suffices to prove that $E\left(\left|x_{s_{n}}\right|\right) \leqq 3 K$ for each $n$. For each $k \in N^{*}$ and $k \leqq n$, let $s_{k}^{\prime}=\max \left(s_{n}, k\right)$. Then $s_{k}^{\prime}$ is a sampling variable and $k \leqq s_{k}^{\prime} \leqq n$. Since

$$
E\left(x_{s^{\prime} k}^{+}\right)=\sum_{j=k}^{n} \int_{\left\{z^{\prime}{ }_{k}=j\right\}} x_{j}^{+} \leqq E\left(x_{n}^{+}\right) \leqq K,
$$

$E\left(x_{s_{k^{\prime}}}\right) \geqq E\left(x_{k}\right)$ by Theorem 3.3. Hence

$$
E\left(\left|x_{z^{\prime} k}\right|\right)=2 E\left(x_{z_{k}^{\prime}}^{+}\right)-E\left(x_{z_{k}^{\prime}}\right) \leqq 2 K-E\left(x_{k}\right) \leqq 3 K .
$$

Letting $k \rightarrow-\infty, E\left(\left|x_{s_{n}}\right|\right) \leqq 3 K$. Thus the proof is complete.

4. Convergence of martingales. For a net $\left(x_{\delta}, \Delta\right)$ of $\mathfrak{F}$-functions the essential supremum, denoted by $\sup _{\delta \in \Delta}^{*} x_{\delta}=y$, is defined to be the smallest (up to

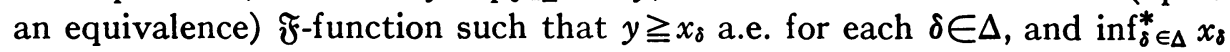
is defined similarly. As usual, we define

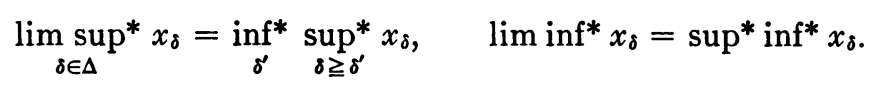

The essential union $\bigcup_{\delta \in \Delta}^{*} K_{\delta}$ of a net of $\mathfrak{F}$-sets is defined to be the smallest (up to an equivalence) $\mathfrak{F}$-set $U$ such that $K_{\delta} \subset U$ a.e. for each $\delta \in \Delta$.

Definition 4.1. A net of $\mathfrak{F}$-sets $\left(K_{\delta}, \Delta\right)$ is called a (or an essential) fine covering of a set $A$ if $K_{\delta} \in \mathfrak{F}_{\delta}$ for each $\delta$, and $A \subset \bigcup_{\delta \geq \delta^{\prime}} K_{\delta}$ (or $A \subset \bigcup_{\delta \geq \delta^{\prime}} K_{\delta}$ a.e.) for each $\delta^{\prime}$. A stochastic basis $\left(\mathfrak{F}_{\delta}, \Delta\right)$ is said to satisfy the Vitali condition $V_{0}$ (or $V_{0}^{*}$ ), if for each (or each essential) fine covering $\left(K_{\delta}, \Delta\right)$ of a set $A$ with $\bar{\mu}(A)<\infty$ there exist $\delta_{i} \in \Delta$ and $\mathfrak{F}_{\delta^{-}}$sets $L_{i} \subset K_{\delta_{i}}, i=1,2, \cdots$, such that $\bar{\mu}\left(A-\bigcup_{i-1}^{\infty} L_{i}\right)=0$, and $L_{i}$ 's are disjoint up to a null set. If the above statement holds only for $\mathfrak{F}_{\text {-sets }} A,\left(\mathfrak{F}_{\delta}, \Delta\right)$ is said to satisfy the condition $V_{1}$. 
The condition $V_{0}^{*}$ is the same as Krickeberg's " $V_{0}$ ". He [8] proves that $V_{0^{\prime}}^{*}$ is satisfied by every linearly ordered stochastic basis $\left(\mathfrak{F}_{\delta}, \Delta\right)$. For $V_{0}$, and even for $V_{1}$, this is not true. For example, let $W$ be the interval $[0,1), \mathfrak{F}$ the class of Lebesgue measurable sets in $[0,1)$, and $\mu$ the Lebesgue measure. Let $\Delta$ be the infinite interval $[0, \infty)$, ordered by the magnitude, and $\mathfrak{F}_{\delta}=\mathfrak{F}$ for every $\delta \in \Delta$. Let $K_{\delta}$ contain only one point $\delta-[\delta]$, where $[\delta]$ is the integral part of $\delta$. Then $\left(K_{\delta}, \Delta\right)$ is a fine covering of $W$. But there does not exist a sequence $\left(\delta_{n}, N\right)$ such that $\mu\left(W-\bigcup_{1}^{\infty} K_{\delta_{n}}\right)=0$. Therefore $\left(\mathfrak{F}_{\delta}, \Delta\right)$ does not have the property $V_{1}$, although $\Delta$ is linearly ordered. However, we have a weaker version of his result as follows:

THEOREM 4.1. Let $\left(\mathfrak{F}_{\delta}, \Delta\right)$ be a countable, linearly-ordered stochastic basis, $\left(K_{\delta}, \Delta\right)$ be a net of $\mathfrak{F}$-sets such that $K_{\delta} \in \mathfrak{F}_{\delta}$ for each $\delta$, and $A$ be a subset of $W$ with $\bar{\mu}(A)<\infty$. If $A \subset \bigcup_{\delta \geq \delta^{\prime}} K_{\delta}$ a.e. for each $\delta^{\prime}$ and $\epsilon>0$, then there exist $\delta_{i} \in \Delta$ and disjoint $\mathfrak{F}_{\delta_{i}}$-sets $L_{i} \subset K_{\delta_{i}}, i=1, \cdots, k$, such that $\bar{\mu}\left(A-\bigcup_{i=1}^{k} L_{i}\right)<\epsilon$.

Proof. Write $\Delta$ as $\left(\delta_{n}, \Delta\right)$ and let $K_{1}^{\prime}=K_{\delta_{1}}$. For $i>1$, define $K_{i}^{\prime}=K_{\delta_{i}}$ $-\bigcup_{j<i} K_{j}^{\prime}$. Then $\left(K_{n}^{\prime}, N\right)$ is a disjoint sequence of $\mathfrak{F}$-sets and $A \subset \bigcup_{n \in N} K_{n}^{\prime}$ a.e. Therefore, $[5$, p. 46$], \bar{\mu}(A)=\sum_{n=1}^{\infty} \bar{\mu}\left(A K_{n}^{\prime}\right)$. Since $\bar{\mu}(A)<\infty$, we can choose $n_{0} \in N$ such that

$$
\bar{\mu}\left(A-\bigcup_{n \leq n_{0}} K_{\delta_{n}}\right)=\bar{\mu}\left(\bigcup_{n>n_{0}} A K_{n}^{\prime}\right)=\sum_{n>n_{0}} \bar{\mu}\left(A K_{n}^{\prime}\right)<\epsilon .
$$

Let $\left(\delta_{n}^{\prime}, n \leqq n_{0}\right)$ be a permutation of $\left(\delta_{n}, n \leqq n_{0}\right)$ such that $\mathfrak{F}_{\delta_{1^{\prime}}} \subset \mathfrak{F}_{\delta_{z^{\prime}}} \subset \ldots$ $\subset \mathfrak{F}_{\delta^{\prime} n_{0}}$, and let $L_{1}=K_{\delta_{1}^{\prime}}$ and $L_{i}=K_{\delta_{i}}-U_{j<i} L_{j}$, for $i>1$. Then $L_{i} \subset K_{\delta^{\prime} i}$, $L_{i} \in \mathfrak{F}_{\delta^{\prime} i}, i=1, \cdots, n_{0}, L_{i}$ 's are disjoint and $\bar{\mu}\left(A-\bigcup_{i \leq n_{0}} L_{i}\right)<\epsilon$.

Lemмa 4.1. Let $\left(x_{\delta}, \Delta\right)$ be a net of $\mathfrak{F}$-functions. Then there exists a countable subset $\Delta^{\prime}$ of $\Delta$ such that

$$
\sup _{\delta \in \Delta}^{*} x_{\delta}=\sup _{\delta \in \Delta^{\prime}} x_{\delta}, \quad \underset{\Delta}{\inf ^{*} x_{\delta}}=\inf _{\Delta^{\prime}} x_{\delta} \text {, a.e. }
$$

This lemma is well known. In the following, we put $\bar{x}=\lim \sup _{\delta \in \Delta} x_{\delta}$, $\tilde{x}=\lim \inf x_{\delta}, \bar{x}^{*}=\lim \sup _{\delta \in \Delta}^{*} x_{\delta}$, and $\tilde{x}^{*}=\lim \inf ^{*} x_{\delta}$.

Lemma 4.2. Let $\left(x_{\delta}, \Delta\right)$ be a net of $\mathfrak{F}$-functions. Then: (i) There exists a countable subdirected set $\Delta^{\prime}$ of $\Delta$ such that

$$
\bar{x}^{*}=\limsup _{\delta \in \Delta^{\prime}} x_{\delta}, \quad \bar{x}^{*}=\lim _{\delta \in \Delta^{\prime}} x_{\delta} \text { a.e. }
$$

(ii) If $\Delta$ has a countable cofinal subset, $\Delta^{\prime}$ may be chosen to be a cofinal subset of $\Delta$.

(iii) If $\Delta$ is linearly ordered, $\Delta^{\prime}$ may be chosen to be a simple subsequence of $\Delta$.

(iv) If $\Delta$ is linearly ordered and has a countable cofinal subset, $\Delta^{\prime}$ may be chosen to be a cofinal simple subsequence of $\Delta$. 
Proof. We may assume that $x_{\delta}^{\prime}$ 's are uniformly bounded and $\mu(W)<\infty$. In fact, if this is not the case, we may consider $x_{\delta}^{\prime}=\arctan x_{\delta}$ and $\mu^{\prime}(V)$ $=\sum_{1}^{\infty}\left(\mu\left(V V_{n}\right) / 1+\mu\left(V_{n}\right)\right) 2^{-n}$, instead of $x_{\delta}$ and $\mu$, where $V_{n}$ is a fixed sequence of disjoint $\mathfrak{F}$-sets such that $\mu\left(V_{n}\right)<\infty$ for each $n$ and $U_{1}^{\infty} V_{n}=W$.

(i) Let $\left(\delta_{n}^{\prime \prime}, N\right)$ be any subset of $\Delta$ and $\left(\delta_{n}, N\right)$ a subset of $\Delta$ such that $\bar{x}^{*}=\inf _{n \geq 1} \sup _{\delta \geq \delta_{n}}^{*} x_{\delta}$ and $\bar{x}^{*}=\sup _{n \geq 1} \inf _{\delta \geq \delta_{n}}^{*} x_{\delta}$ a.e. Choose $\delta_{1}^{\prime}$ and $\left(\delta_{1, n}\right.$; $\left.1 \leqq n \leqq n_{1}\right)$ of $\Delta$ such that $\delta_{1}^{\prime} \geqq \delta_{1}^{\prime \prime}$ and $\delta_{1} ; \delta_{1, n} \geqq \delta_{1}^{\prime}$ for $1 \leqq n \leqq n_{1}$, and

$$
\begin{aligned}
& \mu\left\{\sup _{\delta \geqq \delta^{\prime}}^{*} x_{\delta}>\sup _{n} x_{\delta_{1, n}}+1\right\}<\frac{1}{2}, \\
& \mu\left\{\inf _{\delta \geqq \delta^{\prime} 1} x_{\delta}<\inf _{n} x_{\delta_{1, n}}-1\right\}<\frac{1}{2} .
\end{aligned}
$$

Suppose that $\delta_{i}^{\prime}$ and $\left(\delta_{i, n} ; 1 \leqq n \leqq n_{i}\right)$ of $\Delta$ have been chosen. Then choose $\delta_{i+1}^{\prime}$ and $\left(\delta_{i+1, n} ; 1 \leqq n \leqq n_{i+1}\right)$ of $\Delta$ such that

$$
\begin{aligned}
& \delta_{i+1}^{\prime} \geqq \delta_{i+1}^{\prime \prime}, \delta_{i+1}, \delta_{i, 1}, \delta_{i, 2}, \cdots, \text { and } \delta_{i, n_{i}} \text {, } \\
& \delta_{i+1, n} \geqq \delta_{i+1}^{\prime} \quad \text { for } 1 \leqq n \leqq n_{i+1} \text {; } \\
& \mu\left\{\sup _{\delta \geqq \delta^{\prime} i+1}^{*} x_{\delta}>\sup _{n} x_{\delta_{i+1, n}}+\frac{1}{i+1}\right\}<\frac{1}{2^{i+1}} \text {, } \\
& \mu\left\{\inf _{\delta \geqq \delta^{\prime}+1} x_{\delta}<\inf _{n} x_{\delta_{i+1, n}}-\frac{1}{i+1}\right\}<\frac{1}{2^{i+1}} .
\end{aligned}
$$

Clearly $\delta_{i}^{\prime \prime} \leqq \delta_{i}^{\prime}, \delta_{i} \leqq \delta_{i}^{\prime} \leqq \delta_{i+1}^{\prime}, \delta_{i, j} \leqq \delta_{8}^{\prime} \leqq \delta_{s, t}$ for $i<s$. Then

$$
\begin{aligned}
\bar{x}^{*} & =\inf _{n} \sup _{\delta \geqq \delta_{n}} x_{\delta} \geqq \inf _{n} \sup _{\delta \geqq \delta_{n}^{*}} x_{\delta} \\
& =\lim _{n} \sup _{\delta \leqq \delta_{n}^{\prime}} x_{\delta} \geqq \lim _{i} \sup _{1 \leqq n \geqq n_{i}} x_{\delta_{i, n}} \geqq \lim \sup _{\delta \delta_{1}}^{*} x_{\delta} \\
& \geqq \inf _{\delta^{\prime} \in \Delta} \sup _{\delta \geqq \delta^{\prime}}^{*} x_{\delta}=\bar{x}^{*} \text { a.e. }
\end{aligned}
$$

Let $\Delta^{\prime}=\left(\delta_{i, j} ; i \in N, 1 \leqq j \leqq n_{i}\right)$. We have

$$
\bar{x}^{*}=\lim _{i} \sup _{1 \leqq n \leqq n_{i}} x_{\delta_{i, n}} \leqq \lim _{i} \sup _{\delta \geqq \delta_{i-1}^{\prime}, \delta \in \Delta^{\prime}} x_{\delta} \leqq \lim _{i} \sup _{\delta \geqq \delta_{i}^{\prime}} x_{\delta}=\bar{x}^{*} \text { a.e. }
$$

Therefore

$$
\bar{x}^{*}=\inf _{i} \sup _{\delta \geq \delta_{i-1}^{\prime}, \delta \in \Delta^{\prime}} x_{\delta}=\inf _{\delta \in \Delta^{\prime}} \sup _{\delta \leq \delta^{\prime}, \delta^{\prime} \in \Delta^{\prime}} x_{\delta}=\lim \sup _{\delta \in \Delta^{\prime}} x_{\delta} \text { a.e. }
$$

Similarly,

$$
\tilde{x}^{*}=\liminf _{\delta \in \Delta^{\prime}} x_{\delta} \text { a.e. }
$$

(ii) By taking $\left(\delta_{n}^{\prime \prime}, N\right)$ to be a cofinal subset, $\Delta^{\prime}$ is cofinal. (iii) Since for 
each $i$ we can linearly order $\delta_{i, n}^{\prime}$ for $1 \leqq n \leqq n_{i}, \Delta^{\prime}$ is a simple subsequence. (iv) By taking $\left(\delta_{n}^{\prime \prime}, N\right)$ to be a cofinal subset and ordering $\delta_{i, n}$ for each $i$, $1 \leqq n \leqq n_{i}, \Delta^{\prime}$ is a cofinal simple subsequence.

The part (ii) of this lemma has been proved by Krickeberg [8, p. 334].

From Lemma 4.2, we have immediately:

CoRollary 4.1. Let $\left(x_{\delta}, \Delta\right)$ be a net of $\mathfrak{F}$-functions. If every countable subset $\left(x_{\delta}, \Delta^{\prime}\right)$ converges (may have value $\pm \infty$ ) a.e., or if $\Delta$ is linearly ordered and every simple subsequence converges a.e., then $\left(x_{\delta}, \Delta\right)$ converges essentially. If $\Delta$ is countable, linearly ordered and every cofinal simple subsequence converges a.e., then $\left(x_{\delta}, \Delta\right)$ converges a.e.

The last part has been proved recently by Maharam [11].

LEMMA 4.3. Let $\left(x_{\delta}, \mathfrak{F}_{8}, \Delta\right)$ be a stochastic process satisfying the condition $V_{0}$, or satisfying $V_{1}$ and, in addition, $\bar{x}$ and $\tilde{x}$ are measurable. Then $\bar{x} \leqq \bar{x}^{*}$ and $\tilde{x}^{*} \leqq \tilde{x}$ a.e.

Proof. By Lemma 4.1 there exists a sequence $\left(\delta_{n}, N\right)$ such that $\bar{x}^{*}=\inf _{n} \sup _{\delta \geq \delta_{n}}^{*} x_{\delta}$ a.e. For any real $c$, let $U=\{\bar{x}>c\}$ and $K_{\delta}=\left\{x_{\delta}>c\right\}$. Then $\left(K_{\delta}, \delta \geqq \delta_{n}\right)$ is a fine covering of $U$ for each $n$. By $V_{0}$, or by $V_{1}$ if $\bar{x}$ is measurable, there exist $\delta_{n, k} \geqq \delta_{n}, k=1,2, \cdots$, such that $\bar{\mu}\left\{U-U_{k=1}^{\infty} K_{\delta_{n, k}}\right\}=0$. Hence $\bar{\mu}\left[U-\left\{\sup _{\delta \geqq \delta_{n}}^{*} x_{\delta}>c\right\}\right]=0$, and then $\bar{\mu}\left[U-\bigcap_{n=1}^{\infty}\left\{\sup _{\delta \geqq \delta_{n}}^{*} x>c\right\}\right]=0$. Therefore $\bar{\mu}\left[U-\left\{\inf _{n} \sup _{\delta \geqq \delta_{n}}^{*} x_{\delta} \geqq c\right\}\right]=0$ and $\bar{\mu}\left[U-\left\{\bar{x}^{*} \geqq c\right\}\right]=0$. Hence $\bar{\mu}\left[U_{n-1}^{\infty}\left(\left\{\bar{x}>r_{n}\right\}-\left\{\bar{x}^{*} \geqq r_{n}\right\}\right)\right]=0$, where $\left(r_{n}, N\right)$ is the set of rational numbers. Therefore, for each real number $c,\{\bar{x}>c\} \subset\left\{\bar{x}^{*}>c\right\}$ a.e., and then $\bar{x} \leqq \bar{x}^{*}$ a.e. Similarly, we can prove $\tilde{x}^{*} \leqq \tilde{x}$ a.e.

THEOREM 4.2. Let $\left(x_{\delta}, \mathfrak{F}_{\delta}, \Delta\right)$ be a martingale such that

(4.1) for every $\delta_{0} \in \Delta$ and every $B \in\left(B_{n}, N\right)$ where $B_{n} \in \mathfrak{F}_{\delta_{0}}, \mu\left(B_{n}\right)<\infty$ and $\cup_{1}^{\infty} B_{n}=W$, every subset $\Delta^{\prime}=\left(\delta_{n}, N\right)$ of $\Delta$ with $\delta_{0} \leqq \delta_{1} \leqq \delta_{2} \leqq \cdots$, and every stopping variable $s^{\prime}$ of $\left(\mathfrak{F}_{\delta}, \Delta^{\prime}\right)$, we have $\int_{B\left(s^{\prime} \in \Delta^{\prime}\right)} x_{s^{\prime}}^{+}<\infty$.

(4.2) If $\left(\mathfrak{F}_{\delta}, \Delta\right)$ satisfies the condition $V_{0}$, or satisfies $V_{1}$ and, in addition, $\bar{x}$ and $\tilde{x}$ are measurable, then $\bar{x}=\tilde{x}$ a.e.

$(4.2)^{*}$ If $\left(\mathfrak{F}_{\delta}, \Delta\right)$ satisfies the condition $V_{1}^{*}$, then $\bar{x}^{*}=\tilde{x}^{*}$ a.e.

Proof. We will only give a proof of $(4.2)^{*}$, which is applicable for (4.2) also. Suppose, if possible, that there exist $\alpha$ and $\beta$ such that $\mu\left\{\bar{x}^{*}>\beta>\alpha>\tilde{x}^{*}\right\}$ $>0$. For simplicity we may assume $\beta=1$ and $\alpha=0$. Then there exists an $\mathfrak{F}$-set $U$ contained in some $A \in\left(B_{n}, N\right)$, such that $U \subset\left\{\bar{x}^{*}>1,0>\bar{x}^{*}\right\}$ and $\mu(U)$ $\geqq d>0$. Let $K_{\delta}=A\left\{x_{\delta}>1\right\}$. Then $\left(K_{\delta}, \delta \geqq \delta_{0}\right)$ is an essential covering of the set $U$. By the condition $V_{1}^{*}$, for any $\epsilon>0$, we can find indices $\delta_{i} \geqq \delta_{0}$ and disjoint, up to a null set, $\mathfrak{F}_{\delta_{i}}$-sets $L_{i} \subset K_{\delta_{i}}, i=1, \cdots, k$, such that $\mu\left\{U-A_{1}\right\}<\epsilon$, where $A_{1}=\bigcup_{i=1}^{k} L_{i}$. Choose $\eta_{1} \in \Delta$ such that $\eta_{1} \geqq \delta_{i}, i=1,2, \cdots, k$, and let $k_{\delta}^{\prime}=A_{1}\left\{x_{\delta}<0\right\}$. Then $\left(K_{\delta}^{\prime}, \delta \geqq \eta_{1}\right)$ is an essential fine covering of $U A_{1}$. By the condition $V_{1}^{*}$ again, we can choose $\delta_{i}^{\prime} \geqq \eta_{1}$ and disjoint, up to a null set, 
$\mathfrak{F}_{\delta^{\prime} ;}$ sets $L_{i}^{\prime} \subset K_{\delta_{i}^{\prime}}^{\prime}, i=1, \cdots, k^{\prime}$, such that $\mu\left\{U A_{1}-A_{1}^{\prime}\right\}<\epsilon$, where $A_{1}^{\prime}$ $=\bigcup_{i=1}^{\prime} L_{i}^{\prime}$. Then $A_{1}^{\prime} \subset A_{1}$. Choose $\eta_{1}^{\prime} \in \Delta$ such that $\eta_{1}^{\prime} \geqq \delta_{i}^{\prime}, i=1,2, \cdots, k^{\prime}$. Define $s_{1}$ and $s_{2}$ by:

$$
\begin{aligned}
s_{1}(w) & =\delta_{i} \text { if } w \in L_{i}, 1 \leqq i \leqq k, & s_{2}(w) & =\delta_{i}^{\prime} \text { if } w \in L_{i}^{\prime}, 1 \leqq i \leqq k^{\prime}, \\
& =\eta_{i} \text { if } w \in A-A_{1}, & & \eta_{i} \text { if } w \in A-A_{1}, \\
& =\delta_{0} \text { if } w \in W-A . & & =\eta_{i}^{\prime} \text { if } w \in A_{1}-A_{1}^{\prime}, \\
& & & \delta_{0} \text { if } w \in W-A .
\end{aligned}
$$

Then $s_{1}$ and $s_{2}$ are defined a.e., equivalent to sampling variables, and $s_{1} \leqq s_{2}$ a.e. Since (4.1) implies that $x_{\delta}^{+}$is $\sigma$-summable on $\mathfrak{F}_{\delta^{\prime}}, \delta^{\prime} \in \Delta$, and both $s_{1}$ and $s_{2}$ have only finitely many values. By Theorem $3.5\left(x_{i}^{*}, \mathfrak{F}_{i}^{*}, i=1,2\right)$ is a martingale. By (3.3), $A_{1} \in \mathfrak{F}_{1}^{*}$, and then $\int_{A_{1}} x_{s_{1}}=\int_{A_{1}} x_{s_{2}}$. Therefore

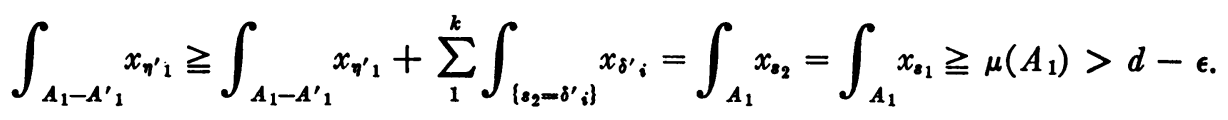

Hence, $\int_{\Lambda_{1}-\Delta_{1}^{\prime}} x_{\eta^{\prime} 1}>d-\epsilon$.

Let $U_{1}=U A_{1}^{\prime}$ and $y_{\delta}=\chi_{A^{\prime}} x_{\delta}$. Then $\left(y_{\delta}, \mathfrak{F}_{\delta}, \delta \geqq \eta_{1}^{\prime}\right)$ is a martingale and satisfies the conditions (4.1) and (4.2) and $U_{1} \subset\left\{\tilde{y}^{*}>1, \tilde{y}^{*}<0\right\}$ and $\bar{\mu}\left(U_{1}\right)$ $>d-\epsilon$. Therefore there exist $\eta_{2}, \eta_{2}^{\prime} \in \Delta$, and sets $A_{2}, A_{2}^{\prime}$ such that $\eta_{2}^{\prime} \geqq \eta_{2}$

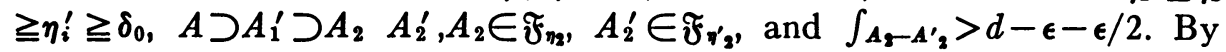
induction, for each positive integer $n$, we can find $\eta_{n}, \eta_{n}^{\prime} \in \Delta$, and sets $A_{n}, A_{n}^{\prime}$ such that $\eta_{n}^{\prime} \geqq \eta_{n} \geqq \eta_{n-1}^{\prime} \geqq \delta_{0}, A \supset A_{n-1}^{\prime} \supset A_{n} \geqq A_{n}^{\prime}, A_{n} \in \mathscr{F}_{\eta_{n}^{\prime}}$ and $\int_{\Lambda_{n}-\Delta_{n}^{\prime} x_{\eta_{n}^{\prime}}}$ $>d-\epsilon-\epsilon / 2-\cdots-\epsilon / 2^{n-1}$.

Define a stopping variable $s^{\prime}$ by $s^{\prime}(w)=\eta_{n}^{\prime}$ if $w \in A_{n-1}^{\prime}-A_{n}^{\prime}$ for $n>1$, and $s^{\prime}(w)=\eta_{1}$ if $w \notin A_{1}$. Then, if $\epsilon$ is small enough,

$$
\int_{A\left\{\varepsilon^{\prime} \in \Delta\right\}} x_{s^{\prime}}^{+} \geqq \sum_{n} \int_{A_{n}-A_{n}^{\prime} n_{n}} x_{\eta_{n}^{\prime}}^{+}=\infty,
$$

which contradicts (4.1). Thus the proof is complete.

In [8], Krickeberg has proven that, if $\left(x_{\delta}, \mathfrak{F}_{\delta}, \Delta\right)$ is an integrable martingale satisfying the condition $V_{0}^{*}$ and

$$
\sup _{\delta} \int_{W} x_{\delta}^{+}<\infty
$$

then $\bar{x}^{*}=\tilde{x}$ a.e. By Theorem 3.7, (4.1) is implied by (4.3). In general, (4.1) is not equivalent to (4.3). To see this, let $W=(0,1], \mathfrak{F}=$ the class of Lebesgue measurable sets in $W$, and $\mu=$ the Lebesgue measure on $W$. For $n \in N$ put $I_{n}=\left(0,1 / 2+1 / 2^{n}\right], \mathfrak{F}_{n}=\otimes\left[I_{k}, k \geqq n\right]$, and $a_{n}=(n-1) 2^{n} /\left(2^{n-1}+1\right)$. Define $x_{1}=0$ and $x_{n}(w)=x_{n-1}(w)$ for $w \notin I_{n-1}, x_{n}(w)=-2^{n}$ for $w \in I_{n-1}-I_{n}$, and $x_{n}(w)=a_{n}$ if $w \in I_{n}$, for $n>1$. Then $\left(x_{n}, \mathfrak{F}_{n}, N\right)$ is a summable martingale which satisfies (4.1) but not (4.3). 
CoRollary 4.2. Let $\left(x_{\delta}, \mathfrak{F}_{\delta}, \Delta\right)$ be a martingale satisfying (4.1). If $V_{0}$ is satisfied by every countable subset $\left(\mathfrak{F}_{\delta}, \Delta^{\prime}\right)$ of $\left(\mathfrak{F}_{\delta}, \Delta\right)$ then $\bar{x}^{*}=\tilde{x}^{*}$ a.e. Therefore, if $\Delta$ is linearly ordered, then $\bar{x}^{*}=\tilde{x}^{*}$ a.e. If $V_{0}$ is satisfied by $\left(\mathfrak{F}_{8}, \Delta\right)$, then $\tilde{x}=\bar{x}=\tilde{x}^{*}=\bar{x}^{*}$ a.e.

This corollary follows immediately from (4.2) and Lemmas 4.2 and 4.3.

Definition 4.2. A stochastic process $\left(x_{\delta}, \mathfrak{F}_{\delta}, \Delta\right)$ is said to satisfy the condition $V_{2}$ (or $V_{2}^{*}$ ), if for $\epsilon>0, \delta_{0} \in \Delta, \alpha, \beta$ reals, and any sets $A, B$ such that $\vec{\mu}(A)<\infty, \bar{\mu}(B)<\infty, A \subset\{\bar{x}>\alpha\}$ and $B \subset\{\bar{x}<\beta\}$ (or $A \subset\left\{\bar{x}^{*}>\alpha\right\}$, $\left.B C\left\{\tilde{x}^{*}<\beta\right\}\right)$, there exist $\delta_{i}, \delta_{j}^{\prime} \in \Delta, \delta_{i} \geqq \delta_{0}, \delta_{j}^{\prime} \geqq \delta_{0}$, and disjoint, up to a null set, $\mathfrak{F}_{\delta_{i}}$-sets $L_{i} \subset\left\{x_{\delta_{i}}>\alpha\right\}, i=1, \cdots, k$, and disjoint, up to a null set, $\mathfrak{F}_{\delta_{j}}^{\prime}$ sets $L_{j}^{\prime} \subset\left\{x_{\delta j}^{\prime}<\beta\right\}, j=1, \cdots, k^{\prime}$, such that $\bar{\mu}\left\{A-\bigcup_{i=1}^{k} L_{i}\right\}<\epsilon, \bar{\mu}\left\{B-\bigcup_{j=1}^{k} L_{j}^{\prime}\right\}$ $<\epsilon$. If the above statement holds only for $\mathfrak{F}_{\text {-sets }} A$ and $B,\left(x_{\delta}, \mathfrak{F}_{\delta}, \Delta\right)$ is said to satisfy the condition $V_{3}$.

LEMma 4.4. If a stochastic process $\left(x_{\delta}, \mathfrak{F}_{\delta}, \Delta\right)$ satisfies the condition $V_{2}$, or the condition $V_{3}$ and $\tilde{x}$ and $\bar{x}$ are measurable then $\bar{x}^{*} \geqq \tilde{x}$ and $\tilde{x} \geqq \tilde{x}^{*}$ a.e. If, moreover, $\Delta$ has a countable cofinal subset, then $\bar{x}^{*}=\bar{x}$ and $\bar{x}=\tilde{x}^{*}$ a.e.

Proof. The first part follows from the proof of Lemma 4.3. If $\Delta$ has a countable cofinal subset, then [8] $\bar{x}^{*} \leqq \bar{x}$ and $\tilde{x} \geqq \tilde{x}^{*}$ a.e., and therefore, $\bar{x}^{*}=\bar{x}$ and $\tilde{x}=\tilde{x}^{*}$ a.e.

CoROllary 4.3. Let $\left(x_{\delta}, \mathfrak{F}_{\delta}, \Delta\right)$ be a martingale satisfying (4.1). If $V_{2}$ is satisfied, or if ' $V_{3}$ is satisfied and $\bar{x}$ and $\bar{x}$ are measurable, then $\bar{x}=\bar{x}$ a.e. If $V_{2}^{*}$ is satisfied, then $\bar{x}^{*}=\tilde{x}$ a.e.

CoROllaRy 4.4. Let $\left(x_{\delta}, \mathfrak{F}_{\delta}, \Delta\right)$ be a semi-martingale satisfying (4.1). If $\left(x_{\delta}, \mathfrak{F}_{\delta}, \Delta\right)$ has the property $V_{2}$, or the property $V_{3}$ and, in addition, $\bar{x}$ and $\bar{x}$ are measurable, and if for any sampling variables $s$ and $s^{\prime}$, which have only finitely many values, and $s \leqq s^{\prime}, E\left(x_{s}\right) \leqq E\left(x_{z^{\prime}}\right)$, then $\bar{x}=\bar{x}$ a.e. Therefore, if $\Delta$ is countable, linearly ordered, and $\left(x_{\delta}, \mathfrak{F}_{\delta}, \Delta\right)$ satisfies $(4.1)$, then $\bar{x}=\tilde{x}$ a.e.

From the definition of terminal separability and Corollary 4.3, we have

COROLlaRy 4.5. Let $\left(x_{\delta}, \mathfrak{F}_{\delta}, \Delta\right)$ be a terminally separable martingale satisfying (4.1) and $V_{3}$. Then $\bar{x}=\bar{x}$ a.e.

Let $T$ be a subset of the real numbers. Then every terminally separable stochastic process $\left(x_{t}, \mathfrak{F}_{t}, T\right)$ satisfies the condition $V_{2}$ by Theorem 4.1. From Corollary 3.3, Theorem 3.7, and Corollary 4.4, we obtain

COROLlaRy 4.6. Let $\left(x_{i}, \mathfrak{F}_{t}, T\right)$ be a terminally separable semi-martingale. If $\sup _{t \in T} E\left(x_{t}^{+}\right)<\infty$, then $\bar{x}=\tilde{x}$ a.e.

When $\bar{\mu}(W)<\infty$ and $\sup _{t \in T} E\left(\left|x_{t}\right|\right)<\infty$, which is equivalent to $\sup _{t \in T} E\left(x_{t}^{+}\right)<\infty$ if $\inf _{t \in T} t \in T$ and $x_{t}$ is summable for each $t$, Corollary 4.6 has proved by Doob $[4 ;$ p. 354$]$. 
If $\left(\mathfrak{F}_{\delta}, \Delta\right)$ satisfies the condition $V_{0}$ or $V_{1}$, then $V_{2}$ or $V_{3}$ is respectively satisfied by every stochastic process $\left(x_{\delta}, \mathfrak{F}_{\delta}, \Delta\right)$. The same relation holds if the $V$ 's are replaced by $V^{*}$ 's. $V_{3}$ and $V_{2}^{*}$ may not be satisfied even by a countable martingale. Dieudonné [3] has an example which is a non-negative bounded martingale in a finite measure space with countable $\Delta$ and $\mu\{\bar{x} \neq \bar{x}\}>0$. Therefore, it does not have the property $V_{3}$ nor $V_{2}^{*}$ by Corollary 4.4.

TheоReм 4.3. Let $\left(x_{n}, \mathfrak{F}_{n}, n \leqq-1\right)$ be an integrable semi-martingale and $E\left(x_{-1}^{+}\right)<\infty$. Let $V \in \cap_{n=-\infty}^{-1} \mathfrak{F}_{n}$ and $0<\mu(V)<\infty$. Then $\lim _{n \rightarrow \infty} x_{n}=x_{\infty}$ exists (may be infinite) a.e. in V.

Proof. Let the restriction of $\mathfrak{F}, \mu, x_{n}$ and $\mathfrak{F}_{n}$ to $V$ be $\mathfrak{F}^{\prime}, \mu^{\prime}, x_{n}^{\prime}$ and $\mathfrak{F}_{n}^{\prime}$ respectively. Then $\left(x_{n}^{\prime}, \mathfrak{F}_{n}^{\prime}, n \leqq-1\right)$ is a semi-martingale defined in a finite measure space $\left(V, \mathfrak{F}^{\prime}, \mu^{\prime}\right)$. Thus $[10$, p. 397$] \lim _{n \rightarrow \infty} x_{n}=x_{\infty}$ exists a.e. in $V$.

The condition $E\left(x_{-1}^{+}\right)<\infty$ cannot be dropped from Theorem 4.3. For example, let $W=(0,1], \mu=$ the Lebesgue measure, and $\mathfrak{F}=\mathfrak{F}_{-1}$ $=\otimes[(0,1 / k], k \geqq 1], \mathfrak{F}_{-n}=\otimes[(0,1 / k], k \geqq n]$. Define $x_{-1}=\infty, x_{-n}=\infty$ if $w \in(0,1 / n]$, and $=1+(-1)^{n}$ if $w \in(1 / n, 1]$. Then $\left(x_{n}, \mathfrak{F}_{n}, n \leqq-1\right)$ is an integrable semi-martingale, but $\bar{x}=2$ and $\tilde{x}=0$ a.e., where $\bar{x}=\lim _{\sup _{n \rightarrow-\infty}} x_{n}$ and $\tilde{x}=\lim \inf _{n \rightarrow-\infty} x_{n}$.

CoROllary 4.8. (i) Let $\left(x_{n}, \mathfrak{F}_{n}, n \leqq-1\right)$ be a semi-martingale and $\sup E\left(\left|x_{n}\right|\right)<\infty$. Then $\lim _{n \rightarrow-\infty} x_{n}=x_{\infty}$ exists a.e. and $E\left(\left|x_{\infty}\right|\right)<\infty$. (ii) If it is a martingale and $E\left(\left|x_{-1}\right|\right)<\infty$, then $\lim x_{n}=x_{\infty}$ exists a.e. and $E\left(\left|x_{\infty}\right|\right)<\infty$.

Proof. For (i), let $\alpha$ and $\beta$ be two arbitrary real numbers and $V=\{\bar{x}>\alpha>\beta>\tilde{x}\}$. If $\alpha>0$, by (3.18) $\mu(V)<\infty$, and if $\beta<0$, by (3.20) $\mu(V)<\infty$. Therefore, $\mu(V)=0$ by Theorem 4.3. Thus $x_{\infty}$ exists a.e. and $E\left(\left|x_{\infty}\right|\right)<\infty$ follows from Fatou's theorem. (ii) follows from (i) immediately, since $\left(\left|x_{n}\right|, \mathfrak{F}_{n}, n \leqq-1\right)$ is a semi-martingale which satisfies the condition of (i).

Another proof of Corollary 4.8 has been given recently by Jerison [7].

The condition $\sup E\left(\left|x_{n}\right|\right)<\infty$ of (i) cannot be replaced by $E\left(\left|x_{n}\right|\right)<\infty$ for each $n \leqq-1$. For example, let $W=[0, \infty), \mu=$ the Lebesgue measure on $W$, and $\mathfrak{F}_{-n}=\circledast[[0, k), k \geqq n]$ and $\mathfrak{F}=\mathfrak{F}_{-1}$. Define $x_{-n}(w)=0$ if $w>2^{n-1}$, and $=-1-\left[1+(-1)^{n-1}\right] / 2$ if $0 \leqq w \leqq 2^{n-1}$. Then $\left(x_{n}, \mathfrak{F}_{n}, n \leqq-1\right)$ is a semimartingale with $E\left(\left|x_{n}\right|\right)<\infty$ for each $n \leqq-1$, but lim $\sup _{n \rightarrow-\infty} x_{n}=-1$ and $\lim \inf x_{n}=-2$ a.e.

The condition $E\left(\left|x_{-1}\right|\right)<\infty$ of (ii) cannot be replaced by $x_{n} \leqq 0$ for each $n \leqq-1$. For example, let $W, \mathfrak{F}_{n}$ and $\mu$ be the same as before, and define $x_{-1}(w)=-2$ for $0 \leqq w \leqq 1, x_{-1}(w)=0$ for $2^{2 n}<w \leqq 2^{2 n+1}$, and $x_{-1}(w)=-3$ for $2^{2 n+1}<w \leqq 2^{2+2}, n=0,1,2, \cdots$. Define $x_{-n}(w)=-1-1-(-1)^{n} / 2$ for $w \leqq 2^{n-1}$ and $x_{-n}(w)=x_{-n+1}(w)$ for $w>2^{n-1}, n=2,3,4, \cdots$. Then $x_{n} \leqq 0$ and $\left(x_{n}, \mathfrak{F}_{n}, n \leqq-1\right)$ is a martingale, but $\bar{x}=-1$ and $\tilde{x}=-2$ a.e. In [7], we can also find another example for the latter. 
TheOREM 4.4. Let $\left(x_{n}, \mathfrak{F}_{n}, N\right)$ be a semi-martingale such that for each $n \in N$,

(4.4) $\mathfrak{F}_{n}$ is generated by disjoint atoms $I_{i}^{n}, i=1,2, \cdots$, of finite measure and $\inf \left[\mu\left(I_{j}^{n+1}\right) / \mu\left(I_{i}^{n}\right): i, j, n, I_{j}^{n+1} \subset I_{i}^{n}\right]=d>0$. If for a given $1>\epsilon>0$, there exist an $\mathfrak{F}$-set $V \subset\left\{\lim \inf x_{n}<K\right\}$ and an $\mathfrak{F}_{r}$-atom $U$ such that

(4.5) $\mu(U V)>(1-\epsilon) \mu(U)$, and for each $n$,

(4.6) $x_{n}(w) \leqq 0$ whenever $w \in U V$, then

(4.7) $\int_{U} x_{r}<K(1-\epsilon / d) \mu(U)$ for $K \leqq 0$ and $\int_{U} x_{r}<K \mu(U)$ for $K \geqq 0$.

Proof. By Theorem 4.1, $\left(\mathfrak{F}_{n}, N\right)$ have the property $V_{0}$. Therefore, there exist disjoint $\mathfrak{F}_{n_{j}}$-atoms $I_{j}, j=1,2, \cdots, s, r<n_{1} \leqq n_{2} \leqq \cdots \leqq n_{s}$, such that $I_{j} \subset U, I_{j} V \neq \varnothing$, and

$$
\mu\left(\bigcup_{j=1}^{\dot{S}} I_{j}\right)>(1-\epsilon) \mu(U) \text { and } x_{n_{j}}(w)<K \quad \text { for } w \in I_{j} .
$$

Let $\mathfrak{P}_{1}=\left(I_{j}, 1 \leqq j \leqq s\right), \mathfrak{A}_{2}=\left(E: E\right.$ is an $\mathfrak{F}_{k}$-atom, $r \leqq k \leqq n_{s}, E \subset U, E V \neq \varnothing$, and $E^{\prime}\left(\bigcup_{j=1}^{\&} I_{j}\right)=\varnothing$ for some $\mathfrak{F}_{k+1}$-atom $\left.E^{\prime} \subset E\right)$, and $\mathfrak{A}_{3}=\mathfrak{A}_{1} \cup \mathfrak{A}_{2}$ then

$$
U=U\left(E: E \in \mathfrak{A}_{3}\right) \text {. }
$$

Suppose, if possible, that it is false. There would exist an $\mathfrak{F}_{n_{2}}$-atom $E_{1} \subset U-U\left(E: E \in \mathfrak{P}_{3}\right)$, and then $E_{1} V=\varnothing$. Let $E_{2} \supset E_{1}$ be an $\mathfrak{F}_{n_{3}-1}$-atom, then $E_{2} V=\varnothing$ and $E_{2}\left(\cup_{1}^{s} I_{j}\right)=\varnothing$, since $E_{2} V \neq \varnothing$ implies $E_{2} \in \mathfrak{A}_{2}$ and $E_{2}\left(U I_{j}\right)_{w} \varnothing$ implies $E_{2}$ contains some $I_{j}$ or vice versa. By induction we could find $\mathfrak{F}_{n_{0}-1^{-}}$ atoms $E_{i}, i=1,2, \cdots, n_{s}-r$, such that $E_{i} V \neq \varnothing$ for each $i$. Now $E_{n_{s}-r} \in F_{r}$ and then $E_{n,-r}=U$, but we have $E_{n_{a}-r}=\varnothing$. Therefore, $U V=\varnothing$, which contradicts (4.5). Thus (4.9) holds.

Since each $\mathfrak{A}_{3}$-set is an $\mathfrak{F}_{i}$-atom for some $r \leqq i \leqq n_{s}$; one of any two nondisjoint $\mathfrak{A}_{3}$-sets is always contained in the other. Hence, we can replace $\mathfrak{A}_{3}$ by $\mathscr{A} \subset \mathfrak{A}_{3}$ which also covers $U$ and consists of disjoint sets only. Let $U_{1}$ be the union of the $\mathfrak{A}$-sets which are also $\mathfrak{P}_{1}$-sets, and let $U_{2}=U-U_{1}$. Then $U_{2}$ is the union of finitely many disjoint $\mathfrak{A}_{2}$-sets, say $C_{i}, i=1,2, \cdots, k$, where $C_{i}$ is an $\mathfrak{F}_{j_{i}}$-atom and $r \leqq j_{i} \leqq j_{i+1} \leqq n_{\text {s. }}$. Since $x_{n}$ is $\sigma$-integrable on each $\mathfrak{F}_{n^{\prime}}, x_{n}$ is integrable in every union of finitely many atoms $I_{j}^{n^{\prime}}$, where $n^{\prime}$ and $j \in N$. Hence

$$
\begin{gathered}
\int_{U} x_{r} \leqq \int_{U} x_{j_{1}}=\int_{C_{1}} x_{j_{1}}+\int_{U-C_{1}} x_{j_{1}} \leqq \int_{C_{1}} x_{j_{1}}+\int_{U-C_{1}} x_{j_{2}} \\
\leqq \int_{C_{1}} x_{j_{1}}+\int_{C_{2}} x_{j_{2}}+\int_{U-\left(C_{1} U C_{2}\right)} x_{j_{2}} \leqq \cdots \leqq \sum \int_{C_{i}} x_{j_{1}}+\int_{U_{1}} x_{n s .}
\end{gathered}
$$

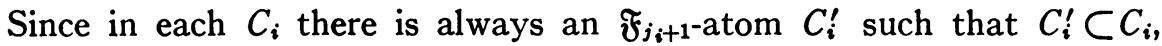
$C_{i}^{\prime} \subset U-U_{1}^{s} I_{j}$, and $\mu\left(C_{i}^{\prime}\right) \geqq d \mu\left(C_{i}\right)$, we have

$$
\mu\left(U-U_{1}\right)=\mu\left(\bigcup_{1}^{k} C_{i}\right) \leqq \mu\left(U-\dot{U}_{1}^{*} I_{j}\right) / d<\epsilon \mu(U) / d
$$


Now $C_{i} V \neq \varnothing$ and $C_{i}$ is an $\mathfrak{F}_{j_{i}}$-atom. By (4.6) we have $\int_{C_{i}} x_{j_{i}} \leqq 0$, for $i=1,2$, $\cdots, k$, and by (4.8) $\int_{U_{1}} x_{n_{\varepsilon}}<K \mu\left(U_{1}\right)$. Thus $\int_{U} x_{r}<K \mu\left(U_{1}\right)<K(1-\epsilon / d) \mu(U)$.

TheOREM 4.5. Let $\left(x_{n}, \mathfrak{F}_{n}, N\right)$ be a semi-martingale satisfying (4.4). Then $\bar{x}=\tilde{x}=x_{\infty}$ exists a.e. in $\{\bar{x}<\infty\}$. If, moreover, $x_{1}>-\infty$ a.e., $x_{\infty}$ is finite a.e. in $\{\bar{x}<\infty\}$.

Proof. Suppose, if possible, that

$$
V^{\prime}=\{\tilde{x}<\bar{x}<\infty\} \text { and } \mu\left(V^{\prime}\right)>0 .
$$

Take $M$ large enough such that $\mu\{\tilde{x}<\tilde{x}<M\}>0$. By replacing $x_{n}$ by $x_{n}-M$, we can assume that $M \leqq 0$. Then there exist numbers $0>\beta>\alpha$ and positive integer $k$ such that $\mu\left\{\bar{x}>\beta>\alpha>\bar{x} ; x_{n}<0\right.$ for $\left.n \geqq k\right\}>0$. We may assume that $k=1$. Since $\mathfrak{F}_{1}$ is $\sigma$-finite, there exists an $\mathfrak{F}$-set $V$ such that

$$
V \subset\left\{\bar{x}>\beta>\alpha>\bar{x} ; x_{n}<0 \text { for } n \in N\right\}, \quad 0<\mu(V)<\infty,
$$

and $V \in \otimes\left[U_{n \in N} \mathfrak{F}_{n}\right]$. For given $1>\epsilon>0$, take $U^{\prime} \in \mathfrak{F}_{r^{\prime}}$ for some $r^{\prime}$ such that

$$
\mu\left(U^{\prime}-V\right)+\mu\left(V-U^{\prime}\right)<\epsilon \mu(V),
$$

and then $\mu\left(U^{\prime} V\right)>(1-\epsilon) \mu\left(U^{\prime}\right)$. Let $A_{n}=U^{\prime}\left\{x_{n}>\beta x_{n}<0\right\}$. Then $\left(A_{n}, n \geqq r^{\prime}\right)$ is a fine covering of $U^{\prime} V$. By the Vitali condition $V_{0}$, there exist disjoint $\mathfrak{F}_{n}$-sets $B_{n} \subset A_{n}, n=1,2, \cdots$, such that $\mu\left(U^{\prime} V-\cup_{n=1}^{\infty} B_{n}\right)=0$. Therefore

$$
\sum_{n=1}^{\infty} \mu\left(B_{n} V\right)=\mu\left(U^{\prime} V\right)>(1-\epsilon) \sum_{n=1}^{\infty} \mu\left(B_{n}\right) \text {. }
$$

Hence, we can choose $r \geqq r^{\prime}$ such that

$$
U\left(B_{r} V\right)>(1-\epsilon) \mu\left(B_{r}\right) .
$$

Therefore there exists an $\mathfrak{F}_{r}$-atom $U \subset B_{r}$ such that

$$
\mu(U V)>(1-\epsilon) \mu(U) \text { and } x_{r}(w)>\beta \text { if } w \in U .
$$

By Theorem 4.4 we have $\int_{U} x_{r}<\alpha(1-\epsilon / d) \mu(U)$, and from (4.13), $\int_{U} x_{r}>\beta \mu(U)$. Thus we have $\beta<\alpha(1-\epsilon / d)$, since $\mu(U)>0$. When $\epsilon$ is small enough, we get a contradiction. Hence, $x_{\infty}$ exists a.e. in $\{\bar{x}<\infty\}$. The second part follows from the following theorem.

THEOREM 4.6. Let $\left(x_{n}, \mathfrak{F}_{n}, N\right)$ be a semi-martingale satisfying (4.4). Then $\lim \sup x_{n}=\bar{x}>-\infty$ a.e., if $x_{1}>-\infty$ a.e.

Proof. To prove this, let us suppose, if possible, that

$$
V^{\prime}=\{\bar{x}=-\infty\} \text { and } \mu\left(V^{\prime}\right)>0 .
$$

As before, we can suppose that there exists an $\mathfrak{F}$-set

$$
V \subset\left\{\bar{x}=-\infty ; x_{n}<0 \text { for } n \in N\right\} \text { and } \infty>\mu(V)>0 \text {, }
$$


and there exists an $\mathfrak{F}_{r}$-set $U^{\prime}$ such that

$$
\mu\left(U^{\prime} V\right)>(1-\epsilon) \mu\left(U^{\prime}\right), \quad \text { for a given } 1>\epsilon>0 .
$$

Clearly there is an $\mathfrak{F}_{r}$-atom $U \subset U^{\prime}$ such that $\mu(U V)>(1-\epsilon) \mu U$. Since $V \subset\{\tilde{x}<K\}$ for every real number $K<0$, by Theorem 4.4 , we have $\int_{U} x_{r}$ $<K \mu(U)$ for every real number $K<0$. Hence, $\int_{U} x_{r}=-\infty$, which contradicts the fact that $x_{1}>-\infty$ a.e. Thus the proof is complete.

5. Applications to derivatives. In this section we are going to show that the classical Lebesgue differentiation theorem of additive functions of bounded variation in a Euclidean space follows from the martingale convergence theorem 4.2. For one dimensional case, it has been given by Doob $[4$, p. 346].

Let $W$ be a $p$-dimensional Euclidean space, $\mu$ the Lebesgue measure in $W$, and $\mathfrak{F}$ the class of Lebesgue measurable sets. A partition $\delta$ is a countable family of disjoint, bounded intervals $I_{k}^{\delta}, k=1,2,3, \cdots$, which are of the form $\left(\alpha_{1}<w_{1} \leqq \beta_{1}, \cdots, \alpha_{p}<w_{p} \leqq \beta_{p}\right)$, such that $\bigcup_{k-1}^{\infty} I_{k}^{\delta}=W . \Delta$ will be the family of all partitions $\delta$ and the order " $\delta<\delta^{\prime \prime}$ of $\Delta$ is defined to mean $\delta^{\prime}$ is a refinement of $\delta$ and every $\delta$-interval is the union of finitely many $\delta^{\prime}$-intervals. For each $\delta \in \Delta, F_{\delta}$ will be the $\sigma$-algebra of sets generated by $I_{k}^{\delta}$ 's. $\Delta_{\alpha}$ will be understood to be the subset of $\Delta$ consisting of all the partitions $\delta$ such that every $\delta$-interval $I^{\delta}$ has parameters of regularity $\left[13\right.$, p. 106] $r\left(I^{\delta}\right) \geqq \alpha>0$.

We need the following lemmas, which are respectively due to Helms. [6] and Krickeberg [8].

LEMMA 5.1. If $\left(x_{\lambda}, \mathfrak{F}_{\lambda}, \Lambda\right)$ is a closed summable martingale in a finite measure space, then it is uniformly integrable and $\left(x_{\lambda}, \Lambda\right)$ converges in mean of order 1.

Lemma 5.2. For every stochastic process $\left(x_{\lambda}, \Lambda\right)$, we have

$$
\int_{\lambda} \liminf _{\lambda}^{*}\left|x_{\lambda}\right| \leqq \lim _{\lambda} \inf \int_{\Lambda}\left|x_{\lambda}\right|
$$

for every $\mathfrak{F}$-set $A$.

Lemma 5.3. Let $\Delta_{a}^{\prime}$ be a subdirected set of $\Delta_{a}$, and let a set $A$ be contained in an $\mathfrak{F}_{\delta_{0}}$-interval $I, \delta_{0} \in \Delta_{\alpha}^{\prime}$. If $\left(K_{\delta}, \Delta_{\alpha}^{\prime}\right)$ is a fine covering of $A$ and $\cup_{\delta \in \Delta^{\prime} \alpha} K_{\delta} \subset I$, then there exist disjoint sets $\left(K_{\delta_{n}}, N\right)$ such that $\delta_{n} \in \Delta_{\alpha}^{\prime}$ and $\bar{\mu}\left(A-\cup_{n=1}^{\infty} K_{\delta_{n}}\right)=0$.

Proof. The proof of this lemma follows closely that of Vitali's Covering Theorem [13, pp. 109-110]. Choose $E_{1}=K_{\delta_{1}}$ for an arbitrary $\delta_{1} \geqq \delta_{0}$, and when the first $n$ disjoint sets $E_{1}=K_{\delta_{1}}, \cdots, E_{n}=K_{\delta_{n}}$ have been defined, we denote $\epsilon_{n}$ the supremum of the diameters of all the sets $K_{\delta}, \delta \geqq \delta_{0}$, which are disjoint from $\bigcup_{i=1}^{n} E_{i}$, and by $E_{n+1}$ any one of these sets with diameter exceeding $\epsilon_{n} / 2$. Since $\Delta_{\alpha}^{\prime}$ is a directed set, such a set must exist, unless $E_{1}, \cdots, E_{n}$ cover the set $A$, in which case we have the proof already. We may, therefore, suppose that this induction can be continued indefinitely.

To show that $\bar{\mu}\left(A-\bigcup_{n=1}^{\infty} E_{n}\right)=0$, let us write $B=A-\bigcup_{n=1}^{\infty} E_{n}$ and suppose, 
if possible, that $\mu(B)>0$. For each $E_{n}$, take a cube $J_{n}$ such that $E_{n} \subset J_{n}$ and $\mu\left(E_{n}\right) \geqq \alpha \mu\left(J_{n}\right)$. Let $J_{n}^{*}$ denote the cube with the same center $J_{n}$ and with diameter $(4 p+1) d\left(J_{n}\right)$, where $d(J)$ is the diameter of the set $J$. Now

$$
\begin{aligned}
\sum_{n=1}^{\infty} \mu\left(J_{n}^{*}\right)=(4 p+1)^{p} \sum_{n=1}^{\infty} \mu\left(J_{n}\right) & \leqq(4 p+1)^{p} \alpha^{-1} \sum_{n=1}^{\infty} \mu\left(E_{n}\right) \\
& \leqq(4 p+1)^{p} \alpha^{-1} \mu(I)<\infty .
\end{aligned}
$$

Therefore, we can find $n_{0} \in N$ such that $\sum_{n_{2} n_{0}} \mu\left(J_{n}^{*}\right)<\mu(B)$. It follows that there exists $x \in B-U_{n \geq n_{0}} J_{n}^{*}$. Take a partition $\delta^{\prime} \geqq \delta_{0}, \delta^{\prime} \in \Delta_{\alpha}^{\prime}$, such that $E_{k}, k=1,2, \cdots, n_{0}$ are $\delta^{\prime}$-intervals. Since $x \in \mathrm{U}_{\delta z \delta^{\prime}} K_{\delta}$, there exists $\delta^{\prime \prime} \geqq \delta^{\prime}$, $\delta^{\prime \prime} \in \Delta_{\alpha}^{\prime}$, such that $x \in K_{\delta^{\prime \prime}} \in \mathfrak{F}_{\delta^{\prime \prime}}$, and $K_{\delta^{\prime \prime}}\left(\bigcup_{k=1} E_{k}\right)=\varnothing$, for the partition $\delta^{\prime \prime}$ is a refinement of $\delta^{\prime}$. Hence $K_{\delta^{\prime \prime}}\left(\mathrm{U}_{k>n_{0}} E_{k}\right) \neq \varnothing$; for, otherwise, we should have, for each $n \in N, 0<d\left(K_{8^{\prime \prime}}\right) \leqq \epsilon_{n} \leqq 2 d\left(E_{n+1}\right) \leqq 2 d\left(J_{n+1}\right)$ and this is clearly impossible since $d\left(J_{n}\right) \rightarrow 0$.

Let $n_{1}$ be the smallest $n$ for which $K_{\delta^{\prime \prime}} E_{n} \neq \varnothing$. Then on the one hand, $K_{8^{\prime \prime}} E_{n}=\varnothing$, for $n=1,2, \cdots, n_{1}-1$, so that

$$
d\left(K_{8^{\prime \prime}}\right) \leqq \epsilon_{n_{1}-1} ;
$$

and on the other hand, $n_{1}>n_{0}$, which implies, by definition of $x$, that $x$ does not belong to $J_{n_{1}}^{*}$. Thus, we find that there are both some points outside $J_{n_{1}}^{*}$ and some points belonging to $E_{n_{1}} \subset J_{n_{1}}$, which are contained in $K_{8^{\prime \prime}}$. Therefore,

$$
d\left(K_{8^{\prime \prime}}\right) \geqq 2 d\left(J_{n_{1}}\right) \geqq 2 d\left(E_{n_{1}}\right)>\epsilon_{n_{1-1}},
$$

in contradiction to (5.3). Thus, $\bar{\mu}(B)=0$, and the proof is complete.

THEOREM 5.1. $\left(\mathfrak{F}_{\delta}, \Delta_{\alpha}^{\prime}\right)$ satisfies the Vitali condition $V_{0}$ for every subdirected set $\Delta_{\alpha}^{\prime}$ of $\Delta_{\alpha}$.

Proof. Let $\left(K_{\delta}, \Delta_{\alpha}^{\prime}\right)$ be a fine covering of a set $A, \bar{\mu}(A)<\infty$, and $\epsilon>0$. Let $\delta_{0} \in \Delta_{\alpha}^{\prime}$. Then there exists a bounded $\mathfrak{F}_{\delta_{0}}$-set $I$ such that

$$
\bar{\mu}(A-I)<\epsilon / 2 \text {. }
$$

Then $\left(I K_{\delta} ; \delta \geqq \delta_{0}, \delta \in \Delta_{\alpha}^{\prime}\right)$ is a fine covering of the set $I A$. By Lemma 5.3, there exists a disjoint sequence $I K_{\delta_{n}}$ such that $\delta_{n} \geqq \delta_{0}, \delta_{n} \in \Delta_{\alpha}^{\prime}$ and

$$
\bar{\mu}\left(I A-\bigcup_{n=1}^{\infty} I K_{\delta_{n}}\right)=0 .
$$

Since $\bar{\mu}\left(\cup_{n-1}^{\infty} A I K_{\delta_{n}}\right)=\sum_{n-1}^{\infty} \bar{\mu}\left(A I K_{\delta_{n}}\right)$ [5, p. 46], there exists $k \in N$ such that

$$
\sum_{n>k} \bar{\mu}\left(A I K_{\delta_{n}}\right)<\epsilon / 2 .
$$

Let $L_{i}=I K_{\delta_{i}}, i=1,2, \cdots, k$, then $L_{i} \subset K_{\delta_{i}}, L_{i} \in F_{\delta_{i}}, L_{i}$ 's are disjoint, and $\bar{\mu}\left(A-\bigcup_{i=1}^{k} L_{i}\right)<\epsilon$. The proof is complete. 
TheOReM 5.2. Let $\left(x_{\delta}, \mathfrak{F}_{\delta}, \Delta_{\alpha}\right)$ be a martingale such that, for every $\delta_{0}$-interval $A$ and every subset $\Delta^{\prime}=\left(\delta_{n}, N\right)$ of $\Delta_{\alpha}$ with $\delta_{0} \leqq \delta_{1} \leqq \delta_{2} \leqq \cdots$,

$$
\sup _{\delta \in \Delta^{\prime}} \int_{A}\left|x_{\delta}\right|<\infty
$$

Then $\lim _{\delta \in \Delta_{\alpha}} x_{\delta}=x_{\infty}(\alpha)$ exists (may be $\pm \infty$ ) a.e. and

$$
x_{\infty}(\alpha)=\lim _{\delta \in \Delta_{\alpha}}^{*} x_{\delta} \text { a.e. }
$$

Proof. The existence of $x_{\infty}(\alpha)$ follows from Theorems 3.7, 4.2, and 5.1. By Corollary 4.2, $x_{\infty}(\alpha)=\lim _{\delta \in \Delta_{\alpha}}^{*} x_{\delta}$ a.e.

Let $f$ be a real valued, finitely additive interval function of bounded variation in $W$ (cf. $[13$, p. 61$]$ ). For $\delta \in \Delta$, define

$$
x_{\delta}(w)=f\left(I_{k}^{\delta}\right) / \mu\left(I_{k}^{\delta}\right) \text { for } w \in I_{k}^{\delta}, \quad k=1,2, \cdots .
$$

Then $\left(x_{\delta}, \mathfrak{F}_{\delta}, \Delta\right)$ is a $\sigma$-summable martingale and $\sup _{\delta \geq \delta_{0}}, \delta \in \Delta \int_{\Delta}\left|x_{\delta}\right|=K(A)<\infty$ for every fixed $\delta_{0}$-interval $A$. Therefore, for each $\alpha>0, \lim _{\delta \in \Delta_{\alpha}} x_{\delta}=x_{\infty}(\alpha)$ exists a.e. and $x_{\infty}(\alpha)=\lim _{\delta \in \Delta_{\alpha}}^{*} x_{\delta}$ a.e. By Lemma 5.2,

$$
\int_{A}\left|x_{\infty}(\alpha)\right| \leqq K(A)<\infty \text {. }
$$

Definition 5.1. Given an interval function $f$, we call the ordinary upper (or lower) Dini derivate of $f$ at $w$, denoted by $\bar{D} f(w)$ (or $\tilde{D} f(w)$ ), the supremum (or infimum) of the number $z$ such that there exists a regular sequence of intervals $I_{n}^{\delta_{n}}$ containing $w$ as the northeastern vertex and tending to $w$ such that $\lim _{n \rightarrow \infty} f\left(I_{n}^{\delta_{n}}\right) / \mu\left(I_{n}^{\delta_{n}}\right)=z$. If $\bar{D} f(w)=\tilde{D}(w)$, the common value will be denoted by $D f(w)$. If, further, $D f(w)$ is finite, $f$ is said to be derivable in the ordinary Dini's sense at $w$ (northeastern).

TheOREM 5.3 (LeBesgue). If $f$ is a real valued, finitely additive interval function of bounded variation, then its ordinary derivative $f^{\prime}$ exists a.e. and $f^{\prime}$ is summable in any bounded interval.

Proof. Let $\bar{D}_{\alpha} f(w)=\lim \sup f\left(I^{\delta}\right) / \mu\left(I^{\delta}\right)$ and $\tilde{D}_{\alpha} f(w)=\lim \inf f\left(I^{\delta}\right) / \mu\left(I^{\delta}\right)$, where $\delta \in \Delta_{\alpha}, I^{\delta}$ is a $\delta$-interval and $w$ is the southeastern vertex of $I^{\delta}$. Let us suppose, if possible, that $\bar{\mu}\{\bar{D} f>\tilde{D} f\}>0$. Then there exists $\beta>0$ such that $\bar{\mu}\left\{\bar{D}_{\beta} f>\tilde{D}_{\beta} f\right\}>0$, since

$$
\bar{D}_{\alpha} f \uparrow \bar{D} f \text { and } \quad \tilde{D}_{\alpha} f \downarrow \tilde{D} f \text { a.e. }
$$

Clearly we have

$$
\limsup _{\delta \in \Delta_{\beta}} x_{\delta} \geqq \bar{D}_{\beta} f \geqq \widetilde{D}_{\beta} f \geqq \lim _{\delta \in \Delta_{\beta}} x_{\delta} \text { a.e. }
$$


Since $\lim _{\delta \in \Delta_{\beta}} x_{\delta}$ exists a.e., $\bar{\mu}\left\{\bar{D}_{\beta} f>\widetilde{D}_{\beta} f\right\}=0$ and then $\bar{D} f=\widetilde{D} f$ a.e. By (5.9) again,

$$
D f=D_{\alpha} f=\lim _{\delta \in \Delta \alpha} x_{\delta} \text { for every } \alpha>0 .
$$

Similarly (5.10) holds for the other $2^{p}-1$ Dini derivatives. Therefore,

$$
f^{\prime}=\lim _{\delta \in \Delta_{\alpha}} x_{\delta}=x_{\infty}(\alpha) \text { a.e. for every } \alpha>0,
$$

and by (5.9) $\int_{A}\left|f^{\prime}\right|<\infty$ for any bounded interval $A$.

Now let $x$ be a $\sigma$-summable point function on every $\mathfrak{F}_{\delta}$ and for each partition $\delta$, define

$$
f\left(I_{k}^{\delta}\right)=\int_{I_{k}^{\delta}} x, \quad I_{k}^{\delta} \in \delta, \quad k=1,2, \cdots
$$

Then $f$ is an additive interval function of bounded variation, and $\left(x_{\delta}, \mathfrak{F}_{\delta}, \Delta\right)$ is a martingale, closed by $x$ on the right. By Theorem 5.3, $f^{\prime}$ exists a.e. and is summable on every bounded interval. By Lemma 5.1, for every bounded interval $A$,

$$
\int_{A} f^{\prime}=\lim _{\delta \in \Delta_{\alpha}} \int_{A} x_{\delta}=\int_{A} x
$$

Therefore, $f^{\prime}=x$ a.e. Thus we have

TheOREM 5.4. An indefinite integral of a $\sigma$-summable function $x$ on every $\mathfrak{F}_{8}$ is differentiable in the ordinary sense a.e., and its ordinary derivative is equal to $x$ a.e.

6. Regular martingale. In the following sections, $R$ will be a subset of the rational numbers, and we will restrict ourselves to the discussion of the semimartingales $\left(x_{r}, \mathfrak{F}_{r}, R\right)$ only.

Definition 6.1. A semi-martingale $\left(x_{r}, \mathfrak{F}_{r}, R\right)$ is said to be regular, if for every sampling process $\left(s_{\lambda}, \Lambda\right),\left(x_{\lambda}^{*}, \Lambda\right)$ is a semi-martingale; to be quasiregular, if for every sampling variable $s, x^{*}$ is $\sigma$-integrable and $E\left(x^{*} \mid \mathfrak{F}_{r}\right)$ $\geqq x_{r}$ a.e. in $\{s \geqq r\}$. A regular semi-martingale $\left(x_{r}, \mathfrak{F}_{r}, R\right)$ is said to be summable, if $\left(x_{\lambda}^{*}, \Lambda\right)$ is summable for every sampling process $\left(s_{\lambda}, \Lambda\right)$. If both $\left(x_{r}, \mathfrak{F}_{r}, R\right)$ and $\left(-x_{r}, \mathfrak{F}_{r}, R\right)$ are regular, quasi-regular, or summable regular semi-martingales, then $\left(x_{r}, \mathfrak{F}_{r}, R\right)$ is said to be a regular, quasi-regular, or summable regular martingale respectively.

There are martingales which are regular semi-martingales, but not regular martingales. For example, let $W=(0,1], \mu=$ the Lebesgue measure on $W$, and $\mathfrak{F}=$ the class of Lebesgue measurable sets. For each $n \in N$, define $x_{n}(w)$ $=-2^{n}$ for $w \in\left(0,2^{-n}\right]$ and $=0$ otherwise; and let $\mathfrak{F}_{n}=B\left[\left(0,2^{-k}\right], k \leqq n\right]$. Then 
$\left(x_{n}, \mathfrak{F}_{n}, N\right)$ is a martingale, which is a regular semi-martingale by Theorem 3.3. Define a sampling variable $s$ by $s(w)=n$ for $w \in\left(0,2^{-n+1}\right]-\left(0,2^{-n}\right]$, $n=1,2, \cdots$. Then $x_{8}=0$. Since $s \geqq 1$ and $E\left(x_{8}\right)=0>E\left(x_{1}\right),\left(x_{n}, \mathfrak{F}_{n}, N\right)$ is not a regular martingale.

The quasi-regularity defined above has been called "regularity" by Snell [14]. In the following we will prove the quasi-regularity is equivalent to the regularity. Thus the regularity defined above is essentially the same as Snell's.

THEOREM 6.1. Let $\left(x_{r}, \mathfrak{F}_{r}, R\right)$ be a semi-martingale. Then it is quasi-regular if, and only if, for sampling variable sof $\left(\mathfrak{F}_{r}, R\right),\left(x_{r}^{*}=x_{\min (8, r)}, \mathfrak{F}_{r}, R\right)$ is a closed semi-martingale by adding $x^{*}=x_{8}$ as the last element.

Proof. Let $\left(x_{r}^{*}, \mathfrak{F}_{r}, R\right)$ be a closed semi-martingale; then $E\left(x^{*} \mid F_{r}\right) \geqq x_{r}^{*}$ a.e. Hence, $\quad \chi_{\{\varepsilon \geq r\}} E\left(x^{*} \mid \mathfrak{F}_{r}\right) \geqq \chi_{\{s \geq r\}} x_{r}^{*}=\chi_{\{s \geq r\}} x_{\min (s, r)}=x_{r}$ a.e. It follows that $\left(x_{r}, \mathfrak{F}_{r}, R\right)$ is quasi-regular. To prove the converse, let $f=\chi_{\{r<r\}}$. For $r^{\prime}>r$. we have

$$
f E\left(x_{r^{\prime}}^{*} \mid \mathfrak{F}_{r}\right)=E\left(f x_{r^{\prime}}^{*} \mid \mathfrak{F}_{r}\right)=E\left(f x_{r}^{*} \mid \mathfrak{F}_{r}\right)=f x_{r}^{*} \text { a.e. }
$$

Since $\min \left(s, r^{\prime}\right)$ is a sampling variable of $\left(\mathfrak{F}_{r}, R\right)$, by the quasi-regularity, we get

$$
(1-f) E\left(x_{r^{\prime}}^{*} \mid \mathfrak{F}_{r}\right) \geqq(1-f) x_{r}=(1-f) x_{r}^{*} \text { a.e. }
$$

Adding (6.1) and (6.2), we have $E\left(x_{r^{\prime}}^{*} \mid \mathfrak{F}_{r}\right) \geqq x_{r}^{*}$ a.e. Therefore, $\left(x_{r}^{*}, \mathfrak{F}_{r}, R\right)$ is a semi-martingale. Now

$$
f E\left(x^{*} \mid \mathfrak{F}_{r}\right)=E\left(f x^{*} \mid \mathfrak{F}_{r}\right)=E\left(f x_{r}^{*} \mid \mathfrak{F}_{r}\right)=f x_{r}^{*} \text { a.e. }
$$

and

$$
(1-f) E\left(x^{*} \mid \mathfrak{F}_{r}\right) \geqq(1-f) x_{r}=(1-f) x_{r}^{*} \text { a.e. }
$$

Hence, $E\left(x^{*} \mid \mathfrak{F}_{r}\right) \geqq x_{r}^{*}$ a.e., and the proof is complete.

When $R=N^{*}$, we have the slightly better result as follows,

THEOREM 6.2. Let $\left(x_{n}, \mathfrak{F}_{n}, N^{*}\right)$ be a semi-martingale, and $s$ be a sampling variable of $\left(\mathfrak{F}_{n}, N^{*}\right)$. Then $E\left(x_{s} \mid \mathfrak{F}_{n}\right) \geqq x_{n}$ a.e. in $\{s \geqq n\}$, if, and only if, $\left(x_{n}^{*}, \mathfrak{F}_{n}, N^{*}\right)$ is a closed semi-martingale by adding $x^{*}$ as the last element.

Proof. The "if" part follows immediately from the previous proof. To prove the "only if" part, we need to check the $\sigma$-integrability of the $x_{n}^{* \prime}$ and to find something to replace (6.2). For $n^{\prime} \leqq n$, let $V \in F_{n^{\prime}}$ such that both $x_{n}$ and $x_{s}$ are integrable in $V$. (a) If $\int_{V} x_{s}^{+}<\infty, \int_{V} x_{n}^{*+}=\int_{V\{s \geq n\}} x_{n}^{+}+\int_{V\{s<n\}} x_{s}^{+}$ $\leqq \int_{V} x_{s}^{+}<\infty$, for the fact that $E\left(x_{s} \mid \mathfrak{F}_{n}\right) \geqq x_{n}$ a.e. in $\{s \geqq n\}$ implies that $E\left(x_{s}^{+} \mid \mathfrak{F}_{n}\right) \geqq x_{n}^{+}$a.e. in $\{s \geqq n\}$, by Corollary 3.2. (b) If 


$$
\begin{aligned}
\int_{V} x_{n}^{+}<\infty, \int_{V} x_{n}^{*+}=\int_{V\{\varepsilon \geq n\}} x_{n}^{+} \int_{V\{\varepsilon<n\}} x_{s}^{+} & \leqq \int_{V(\bullet \geq n\}} x_{n}^{+}+\sum_{j<n} \int_{V\{\varepsilon=j\}} x_{j}^{+} \\
& \leqq \int_{V\{\bullet \geq n\}} x_{n}^{+}+\sum_{j<n} \int_{V\{e-j\}} x_{n}^{+} \\
& \leqq \int_{V} x_{n}^{+}<\infty .
\end{aligned}
$$

(c) If both $\int_{V} x_{s}^{-}<\infty$ and $\int_{V} x_{n}^{-}<\infty$, then

$$
\int_{V} x_{n}^{*-}=\int_{V\{\bullet \geq n\}} x_{n}^{-}+\int_{V\{a<n\}} x_{s}^{-} \leqq \int_{V} x_{n}^{-}+\int_{V} x_{\varepsilon}^{-}<\infty .
$$

Hence, $x_{n}^{*}$ is $\sigma$-integrable on every $\mathfrak{F}_{n^{\prime}}, n^{\prime} \leqq n$. Therefore, $x_{n}^{*}$ is $\sigma$-integrable on every $\mathfrak{F}_{n^{\prime}}, n^{\prime} \in N^{*}$. To replace $(6.2)$, let $f^{\prime}=\chi_{\{s=n\}}$, and $f^{\prime \prime}=\chi_{\{s>n\}}$. Then

$$
\begin{aligned}
\left(f^{\prime}+f^{\prime \prime}\right) E\left(x_{n+1}^{*} \mid \mathfrak{F}_{n}\right) & =E\left(f^{\prime} x_{n}+f^{\prime \prime} x_{n+1} \mid \mathfrak{F}_{n}\right) \geqq f^{\prime} x_{n}+f^{\prime \prime} x_{n} \\
& =\left(f^{\prime}+f^{\prime \prime}\right) x_{n}^{*} \text { a.e. }
\end{aligned}
$$

Thus $E\left(x_{n+1}^{*} \mid \mathfrak{F}_{n}\right) \geqq x_{n}^{*}$ a.e., since $f E\left(x_{n+1}^{*} \mid \mathfrak{F}_{n}\right)=f x_{n}^{*}$ a.e. as proved in (6.1) before.

TheOREM 6.3. A semi-martingale $\left(x_{r}, \mathfrak{F}_{r}, R\right)$ is quasi-regular, if, and only if it is regular.

Proof. Let $\left(x_{r}, \mathfrak{F}_{r}, R\right)$ be a semi-martingale and $s$ be a sampling variable of $\left(\mathfrak{F}_{r}, R\right)$. For $r \leqq r^{\prime}$ define $S_{r}=r$, and define $s_{\infty}=\max \left(s, r^{\prime}\right)$. Let $R^{\prime}=(t: t \in R$ and $t \leqq r^{\prime}$, or $\left.t=\infty\right)$, and $f=\chi_{\left\{s \geq r^{\prime}\right\}}$. Then $\left(s_{r}, R\right)$ is a sampling process of $\left(\mathfrak{F}_{r}, R\right)$. If $\left(x_{r}, \mathfrak{F}_{r}, R\right)$ is regular,

$$
\begin{aligned}
f E\left(x^{*} \mid \mathfrak{F}_{r^{\prime}}\right) & =E\left(f x^{*} \mid \mathfrak{F}_{r^{\prime}}\right)=E\left(f x_{\varepsilon} \mid \mathfrak{F}_{r^{\prime}}\right)=E\left(f x_{\infty}^{*} \mid \mathfrak{F}_{r^{\prime}}\right) \\
& =f E\left(x_{\infty}^{*} \mid \mathfrak{F}_{r^{\prime}}\right) \geqq f x_{r^{\prime}}^{*}=f x_{r^{\prime}} \text { a.e. }
\end{aligned}
$$

Therefore $\left(x_{r}, \mathfrak{F}_{r}, R\right)$ is quasi-regular. To prove the converse, let $\left(x_{r}, \mathfrak{F}_{r}, R\right)$ be quasi-regular and $\left(s_{\lambda}, \Lambda\right)$ be a sampling process of $\left(\mathfrak{F}_{r}, R\right)$. It suffices to prove that, for an $\mathfrak{F}_{\lambda}^{*}$-set $V$, in which $x_{\lambda}^{*}$ is integrable, and $\lambda<\lambda^{\prime}$ we have

$$
\int_{V} x_{\lambda}^{*} \leqq \int_{V}^{x_{\lambda^{\prime}}^{*}}
$$

If $U=V\left\{s_{\lambda}=r\right\}, U \in \mathfrak{F}_{r}$ by Corollary 3.1. Hence $\int_{U} x_{\lambda^{\prime}}^{*}=\int_{U} E\left(x_{\lambda^{\prime}}^{*} \mathfrak{F}_{r}\right) \geqq \int_{U} x_{r}$ $=\int_{U} x_{\lambda}^{*}$. Therefore, $(6.3)$ is true. Hence, $\left(x_{\lambda}^{*}, \mathfrak{F}_{\lambda}^{*}, \Lambda\right)$ is a semi-martingale.

COROLlaRy 6.1. If $\left(x_{r}, \mathfrak{F}_{r}, R\right)$ is a regular semi-martingale, then $\left(x_{\lambda}^{*}, \mathfrak{F}_{\lambda}^{*}, \Lambda\right)$ is a semi-martingale for every sampling process $\left(s_{\lambda^{\prime}}, \Lambda\right)$ of $\left(\mathfrak{F}_{r}, R\right)$. 
Proof. Let $\left(x_{r}, \mathfrak{F}_{r}, R\right)$ be a regular semi-martingale; then it is quasiregular, by Theorem 6.3. Therefore, by the last line of the previous proof, we have that $\left(x_{\lambda}^{*}, \mathfrak{F}_{\lambda}^{*}, \Lambda\right)$ is a semi-martingale.

THEOREM 6.4. Let $\left(x_{r}, \mathfrak{F}_{r}, R\right)$ be a semi-martingale such that

$$
\lim \sup _{r} \int_{(0>r)} x_{r}^{+}<\infty \text { and } \liminf \int_{(b>r)} x_{r}^{+}=0,
$$

for every sampling variable s. (i) If $E\left(x_{r}^{+}\right)<\infty$ for each $r \in R$, then $\left(x_{r}, \mathfrak{F}_{r}, R\right)$ is regular and $E\left(x_{s}^{+}\right)<\infty$ for every s. (ii) If $R=N$ and $E\left(x_{n}^{-}\right)<\infty$ for each $n \in N$, then $\left(x_{n}, \mathfrak{F}_{n}, N\right)$ is regular and $E\left(x_{s}^{-}\right)<\infty$. Conversely, let $\left(x_{r}, \mathfrak{F}_{r}, R\right)$ be a regular semi-martingale and $E\left(x_{s}^{+}\right)<\infty$ for every sampling variable s, then

$$
\lim _{r} \int_{(0>r)} x_{r}^{+}=0 \text {. }
$$

Proof. If (6.4) is satisfied and $E\left(x_{r}^{+}\right)<\infty$ for each $r$, then $E\left(x_{r}^{+}\right)<\infty$ for every sampling variable $s$ of $\left(\mathfrak{F}_{r}, R\right)$ by Lemma 3.1 , and $\left(x_{r}, \mathfrak{F}_{r}, R\right)$ is regular by Theorem 3.3. If $R=N, E\left(x_{n}^{+}\right)<\infty$ for each $n \in N$, and (6.4) is satisfied, then $E\left(x_{s}^{-}\right)<\infty$ for every sampling variable $s$ of $\left(\mathfrak{F}_{n}, N\right)$ by Lemma 3.2, and $\left(x_{n}, \mathfrak{F}_{n}, N\right)$ is regular by Theorem 3.3 again. To prove the last part, let $s$ be a sampling variable of $\left(\mathfrak{F}_{r}, R\right)$. Then $E\left(x_{s}^{+} \mid \mathfrak{F}_{r}\right) \geqq x_{r}^{+}$a.e. in $\{s \geqq r\}$ by Theorem 6.3. Therefore

$$
\lim _{r} \int_{(8>r)} x_{r}^{+} \leqq \lim _{r} \int_{(\diamond>r)} x_{r}^{+}=0 .
$$

7. Set functions. Let $\$$ be an algebra of $\mathfrak{F}$-sets and $M(\mathbb{S})$ be the class of all real-valued, bounded, finitely additive set functions on $\mathbb{B}$. The subset of $M(\mathbb{S})$ consisting of non-negative functions will be denoted by $M^{+}(\mathbb{S})$. A set function $f \in M^{+}(\mathbb{S})$ is said to be purely finitely additive, if zero is the only countably additive, bounded set function $g$ such that $0 \leqq g \leqq f$. A set function $f \in M(\mathbb{S})$ is said to be purely finitely additive, if both its positive part $f^{+}$and negative part $f^{-}$are purely finitely additive, where

$$
f^{+}(V)=\sup \{f(U): U \subset V, U \in \mathbb{S}\} \text { for } V \in \mathbb{B} \text { and } f^{-}=(-f)^{+} \text {. }
$$

Yosida and Hewitt [15] proved that, for every $f \in M(\mathbb{B})$, there exists a unique decomposition

$$
f=f_{p}+f_{c},
$$

where $f_{p}$ is purely finitely additive and $f_{c}$ is countably additive. If $\&$ is a $\sigma$-algebra, then $f_{c}$ can be further uniquely written as

$$
f_{c}=f_{\mathrm{s}}+f_{a},
$$


where $f_{s}$ and $f_{a}$ are respectively the singular and absolutely continuous parts (relative to $\mu$ ) of $f$.

Let $\mathscr{S}_{1} \subset{S_{2}}_{2} \subset{S_{3}}_{3} \subset \cdots$ be a sequence of $\sigma$-algebras of $\mathfrak{F}$-sets, and $\mathbb{B}$ be

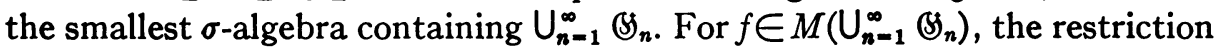
of $f$ to $\mathcal{B S}_{n}$ is denoted by $f_{n}$. We also denote the purely finitely additive, the singular, and the absolutely continuous parts of $f_{n}$ respectively by $f_{n p}, f_{n s}$, and $f_{n a}$. If $\bar{f}_{s}$ and $\vec{f}_{a}$ are the singular and the absolutely continuous parts of the ex-

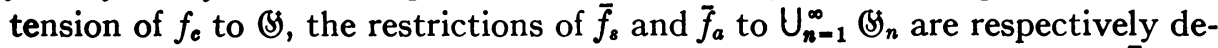
noted by $f_{c}$ and $f_{a}$ : Let $x$ and $x_{n}$ be the Radon-Nikodym derivatives of $\bar{f}_{a}$ and $f_{n a}^{\prime}$. Andersen and Jessen [1] proved that $\lim x_{n}=x$ a.e., if $f_{p}=0$. Theorem 7.1 will extend their theorem to the general case.

Lemma 7.1. Let $f \in M^{+}\left(\cup_{n-1}^{\infty} \mathfrak{S}_{n}\right)$ and $f=f_{p}$. Then $\lim x_{n}=0$ a.e.

Proof. Clearly, $\left(-x_{n}, \mathfrak{S}_{n}, N\right)$ is a semi-martingale and $E\left(x_{n}\right) \leqq f(W)$. By Corollary 4.3, $\lim x_{n}=x_{\infty}$ exists a.e. Let $V \in \cup_{n-1}^{\infty} \mathfrak{S}_{n}$. Since $f_{p}(V)=f(V)$ $\geqq \lim \int_{V} x_{n}, f_{p}(V) \geqq \int_{V} x_{\infty}$, by Fatou's theorem. By the definition of purely finite additivity, $\int_{V} x_{\infty}=0$ for every $V \in \cup_{n-1}^{\infty}\left(S_{n}\right.$. Since $\$=B\left(\bigcup_{n=1}^{\infty} \oiint_{n}\right)$ and $x_{\infty}$ is (5)-measurable, $x_{\infty}=0$ a.e.

ThEOREM 7.1. Let $f \in M\left(\cup_{n=1}^{\infty} \mathcal{G}_{n}\right)$. Then $\lim x_{n}=x$ a.e.

Proof. We may assume that $f \in M^{+}\left(\bigcup_{n=1}^{\infty}\left(\mathcal{S}_{n}\right)\right.$. Let $f^{\prime}=f_{c}$ and $x_{n}^{\prime}$ and $x_{c n}$ be respectively the Radon-Nikodym derivatives of the absolutely continuous parts of the restrictions of $f^{\prime}$ and $f_{c}$ to $\mathcal{B}_{n}$. Then $x_{n}{ }^{\prime}=x_{n}-x_{c n}$ and $\lim x_{n}{ }^{\prime}=0$ by Lemma 7.1, since $f^{\prime}$ is purely finitely additive. By Andersen and Jessen's theorem, $\lim x_{c n}=x$ a.e. Therefore $\lim x_{n}=x$ a.e.

Let $\left(x_{n}, \mathfrak{F}_{n}, N\right)$ by a semi-martingale and $\sup _{n} E\left(\left|x_{n}\right|\right)<\infty$. Define

$$
f(V)=\lim _{n} \int_{V} x_{n} \text { for } V \in \bigcup_{1}^{\infty} \mathfrak{F}_{n} .
$$

Then $f \in M\left(\bigcup_{1}^{\infty} \mathfrak{F}_{n}\right)$.

THEOREM 7.2. If $\left(x_{n}, \mathfrak{F}_{n}, N\right)$ is a martingale and $\sup _{n} E\left(\left|x_{n}\right|\right)<\infty$ then $f$ is completely additive if, and only if, $\left(x_{n}, \mathfrak{F}_{n}, N\right)$ is regular.

Proof. Let $\left(x_{n}, \mathfrak{F}_{n}, N\right)$ be regular and $V_{n}$ be a sequence of disjoint sets such that

$$
V_{n} \in \mathfrak{F}_{n}, \quad \bigcup_{1}^{\infty} V_{n}=V \in \mathfrak{F}_{n_{0}} \text { for some } n_{0}
$$

Define a sampling variable $s$ by

$$
\{s=n\}=V_{n} \text { for } n<n_{0} \text { and } \quad\left\{s=n_{0}\right\}=(W-V) \cup \bigcup_{1}^{n_{0}} V_{n} .
$$

Then for $N>n_{0}$ 


$$
f(V)-\sum_{1}^{N} f\left(V_{n}\right)=f\left(\bigcup_{n+1}^{\infty} V_{n}\right)=\int_{\bigcup_{n+1}^{\infty} V_{n}} x_{N}=\int_{[0>N\}} x_{N}=\int_{\{0>N\}} x_{s} .
$$

Therefore

$$
\lim _{N}\left|f(V)-\sum_{1}^{N} f\left(V_{n}\right)\right| \leqq \lim _{N} \int_{(\bullet>N)}\left|x_{z}\right|=0,
$$

by Theorems 6.2 and 3.7. To prove the converse, let $f$ be completely additive and $s$ a sampling variable. For fixed $n$, put

$$
V=\left\{E\left(x_{s} \mid \mathfrak{F}_{n}\right)<x_{n}, s \geqq n\right\} \quad \text { and } \quad V_{k}=V \cdot\{s=k\} \quad \text { for } k \geqq n .
$$

Then $V \in \mathfrak{F}_{n}, V_{k} \in \mathfrak{F}_{k}$ and $V=\bigcup_{n}^{\infty} V_{k}$. Therefore $\sum_{n}^{\infty} f\left(V_{k}\right)=f(V)=\int_{V} x_{n}$ $\geqq \int_{V} x_{s}=\sum_{n}^{\infty} \int_{V_{k}} x_{k}=\sum_{n}^{\infty} f\left(V_{k}\right)$.

$$
\int_{V} x_{n}=\int_{V} x_{z}
$$

Hence

$$
\mu(V)=0 .
$$

Similarly, we can prove that $\mu\left\{E\left(x_{s} \mid \mathfrak{F}_{n}\right)>x_{n}, s \geqq n\right\}=0$. Thus $\left(x_{n}, \mathfrak{F}_{n}, N\right)$ is quasi-regular. By Theorem $6.3\left(x_{n}, \mathfrak{F}_{n}, N\right)$ is regular.

THEOREM 7.3. Suppose that $\left(x_{n}, \mathfrak{F}_{n}, N\right)$ be a semi-martingale and that $\sup _{n} E\left(\left|x_{n}\right|\right)<\infty$. If $f$ is completely additive, then $\left(x_{n}, \mathfrak{F}_{n}, N\right)$ is regular. Conversely, if $\left(x_{n}, \mathfrak{F}_{n}, N\right)$ is regular and if

$$
\lim _{n} \int_{\{8>n\}} x_{n}^{-}=0
$$

for every sampling variable $s$, then $f$ is completely additive.

Proof. Put $x_{n}^{\prime}=x_{n}-\sum_{1}^{n} d_{j}$ where $d_{1}=0$ and $d_{j}=E\left(x_{j} \mid \Im_{j-1}\right)-x_{j-1}$ for $j>1$. Then $d_{j} \geqq 0$ and $\left(x_{n}^{\prime}, \mathfrak{F}_{n}, N\right)$ is a summable martingale [4, p. 297]. Hence

$$
E\left(x_{n}^{\prime}\right)=E\left(x_{1}^{\prime}\right)=E\left(x_{1}\right),
$$

that is,

$$
E\left(x_{n}\right)=E\left(x_{1}\right)+\sum_{1}^{n} E\left(d_{j}\right)
$$

Therefore

$$
\sum_{1}^{n} E\left(d_{j}\right)<\infty \text { and } \sup _{n} E\left(\left|x_{n}^{\prime}\right|\right)<\infty
$$

For $V \in \bigcup_{1}^{\infty} \mathfrak{F}_{n}$, put 


$$
f^{\prime}(V)=\lim _{n} \int_{V} x_{n}^{\prime} \quad \text { and } \quad g(V)=\lim _{n} \int_{V} \sum_{1}^{n} d_{j} .
$$

Then $g$ is completely additive. Suppose that $f$ be completely additive, then $f^{\prime}$ is completely additive. By Theorem $7.2,\left(x_{n}{ }^{\prime}, \mathfrak{F}_{n}, N\right)$ is a regular martingale. Hence $\left(x_{n}, \mathfrak{F}_{n} N\right)$ is a regular semi-martingale. Conversely, let $\left(x_{n}, \mathfrak{F}_{n}, N\right)$ be regular and $s$ be a sampling variable. Then

$$
\begin{aligned}
& \lim _{\bar{n}} \int_{\{8>n\}} x_{n}^{\prime+} \leqq \lim _{n} \int_{\{8>n\}} x_{n}^{+}=0 . \\
& \lim _{n} \int_{\{8>n\}} x_{n}^{\prime-} \leqq \lim _{n}\left(\int_{\{8>n\}} x_{n}^{-}+\sum_{1}^{n} d_{j}\right)=0 .
\end{aligned}
$$

Therefore $\left[4\right.$, p. 302] $\left(x_{n}^{\prime}, \mathfrak{F}_{n}, N\right)$ is a regular martingale. By Theorem 7.2, $f^{\prime}$ is completely additive. Therefore $f$ is completely additive.

The condition (7.4) is necessary in the following sense: there exist regular semi-martingales $\left(x_{n}, \mathfrak{F}_{n}, N\right)$ such that $\sup _{n} E\left(\left|x_{n}\right|\right)<\infty$, but $f$ are not completely additive. In fact, the example in $\$ 6$ is one of them.

In concluding this section, let $W$ be space of all real-valued sequences, $\left(x_{n}, N\right)$ the sequence of coordinate variables, and $\mathfrak{F}_{n}=B\left(x_{1}, \cdots, x_{n}\right)$. Then Moy [12], see also $\left[10\right.$, p. 94] proves that if $\left(x_{n}, \mathfrak{F}_{n}, \mu\right)$ is a martingale then the necessary and sufficient condition for $f$ to be completely additive is $\sup _{n} E\left(\left|x_{n}\right|\right)<\infty$. Thus by Theorem 7.2 we have:

THEOREM 7.4. Suppose that $\left(x_{n}, N\right)$ be the sequence of coordinate variables of the space $W$ of all real-valued sequences, $\mathfrak{F}_{n}=\mathbb{B}\left(x_{1}, \cdots, x_{n}\right)$, and $\left(x_{n}, \mathfrak{F}_{n}, N\right)$ be a martingale. If $\sup _{n} E\left(\left|x_{n}\right|\right)<\infty$, then $\left(x_{n}, \mathfrak{F}_{n} N\right)$ is regular.

\section{BIBLIOGRAPHY}

1. E. S. Andersen and B. Jessen, Some limit theorems on set-functions, Danske Vid. Selsk. Mat.-Fys. Medd. vol. 25 no. 5 (1948) pp. 1-8.

2. S. Bochner, Partial ordering in the theory of martingales, Ann. of Math. vol. 62 (1955) pp. $162-169$.

3. J. Dieudonné, Sur un theorème de Jessen, Fund. Math. vol. 37 (1950) pp. 242-248.

4. J. L. Doob, Stochastic processes, New York, Wiley, 1953.

5. P. R. Halmos, Measure theory, New York, Van Nostrand, 1950.

6. L. L. Helms, Mean convergence of martingales, Trans. Amer. Math. Soc. vol. 87 (1958) pp. $439-446$.

7. M. Jerison, Martingale formulation of ergodic theorems, Proc. Amer. Math. Soc. vol. 10 (1959) pp. 531-539.

8. K. Krickeberg, Convergence of martingales with a directed index set, Trans. Amer. Math. Soc. vol. 83 (1956) pp. 313-337. 486.

9. - Stochastische Konvergenz von Semimartingalen, Math. Z. vol. 66 (1957) pp. 470-

10. M. Loève, Probability theory, New York, Van Nostrand, 1955. 
11. D. Maharam, On two theorems of Jessen, Proc. Amer. Math. Soc. vol. 9 (1958) pp. 995-999.

12. S. T. C. Moy, Measure extensions and the martingale convergence theorem, Proc. Amer. Math. Soc. vol. 4 (1953) pp. 902-907.

13. S. Saks, Theory of the integral, Warszawa-Lwòw, 1937. Reprinted Stechert-Hafner Publishing Company, New York.

14. J. L. Snell, Applications of martingale system theorems, Trans. Amer. Math. Soc. vol. 73 (1952) pp. 293-312.

15. K. Yosida and E. Hewitt, Finitely additive measures, Trans. Amer. Math. vol. 72 (1952) pp. 46-66.

iBM Research Center,

YORKTOWN HEIGHTS, NEW YORK 